Bo Chen

Marco Serena

\title{
Bid Caps and Disclosure Policies
}

Max Planck Institute for Tax Law and Public Finance

Working Paper 2020 - 08

June 2020

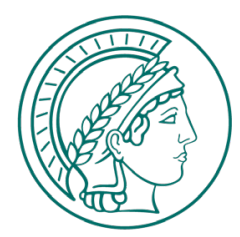

Max Planck Institute for

Tax Law and Public Finance

Department of Business and Tax Law

Department of Public Economics

http:/ / www.tax.mpg.de 
Working papers of the Max Planck Institute for Tax Law and Public Finance Research Paper Series serve to disseminate the research results of work in progress prior to publication to encourage the exchange of ideas and academic debate. Inclusion of a paper in the Research Paper Series does not constitute publication and should not limit publication in any other venue. The preprints published by the Max Planck Institute for Tax Law and Public Finance represent the views of the respective author(s) and not of the Institute as a whole. Copyright remains with the author(s).

Max Planck Institute for Tax Law and Public Finance

Marstallplatz 1

D-80539 Munich

Tel: $\quad+498924246-0$

Fax: $\quad+498924246-501$

E-mail: ssrn@tax.mpg.de

http://www.tax.mpg.de 


\title{
Bid Caps and Disclosure Policies*
}

\author{
Bo Chen ${ }^{\dagger}$ and Marco Serena ${ }^{\ddagger}$
}

July 2020

\begin{abstract}
We examine the effect of publicly disclosing or concealing bidders' types in an all-pay auction with a common bid cap. We call partial (full) disclosure policy the setup where the contest designer's disclosure policy is (not) contingent on type realization. Despite a bid cap possibly increasing the expected expenditures under a partial disclosure policy and the expected expenditures of low-types under a policy of full disclosure, the bid cap unambiguously decreases the expected total expenditures, regardless of the disclosure policy. For any given bid cap and allowing for full and partial disclosure policies, we find that expenditures are minimized with the policy in which a designer discloses bidders' types only if both players have a low valuation of the prize. The expenditures are maximized with the full concealment policy or with the policy in which a designer discloses the information only if both players have a high valuation. All in all, when the designer can choose both the disclosure policy and the bid cap, revenue is maximized employing full concealment with no bid cap.
\end{abstract}

Keywords: contest, all-pay auction, stochastic abilities, bid cap, disclosure. JEL classification: C72, D44, D82.

${ }^{*}$ We thank Bo Chen [Southern Methodist University (SMU)] and Jingfeng Lu for comments and discussions. A part of the paper was written when the first author was serving SMU. Chen is grateful for the support of SMU. Remaining errors are ours. Declarations of interest: none.

†Shenzhen-Audencia Business School, China (bochenbonn@gmail.com).

${ }^{\ddagger}$ Max Planck Institute for Tax Law and Public Finance, Germany (marco.serena85@gmail.com). 


\section{Introduction}

Lobbyists spend resources to influence legislations and political processes. In doing so, they often compete with other lobbyists. Lobbyists may expand higher expenditures in close races, when they are similarly "strong" in their influence strength, than in case of a substantial difference in influence strengths. This conventional insight about expenditures and asymmetries among lobbyists is the key force behind two parallel strands of the literature.

The first strand of the literature investigates the effect on lobbyists' expenditures of a cap in the expenditures itself (e.g., Che and Gale, 1998; Gavious et al., 2002; Sahuguet, 2006; Szech, 2015; Einy et al., 2016; Chen, 2019b; Olszewski and Siegel, 2019). Under linear cost function, in the ex-ante asymmetric setup of Che and Gale (1998), a cap in expenditures may increase total expenditures by constraining the stronger lobbyist and in turn giving hope to the weaker lobbyist (leveling the playing-field), while in the ex-ante symmetric setup of Gavious et al. (2002) a cap always decreases total expenditures as the leveling-the-playing-field effect is absent. ${ }^{1}$

The second strand of the literature analyzes how the information that competing lobbyists know about each other shapes the lobbyists' expenditures (e.g., Fu et al., 2014; Zhang and Zhou, 2016; Lu et al., 2018; Serena, 2019). A policy maker may leverage on the fact that lobbyists may a priori not be aware of the set of other competitors, and strategically fine-tune the disclosure of information, so as to affect expenditures. Such a policy maker choosing the disclosure policy will face an ex-ante trade-off between contingencies when competing lobbyists are symmetric (which yield high expenditures) and the contingencies when competing lobbyists are asymmetric (which yield low expenditures).

The two above strands of the literature build on the same key conflicting force between symmetry/asymmetry among lobbyists, though leveraging on two completely different tools: caps and information. Nevertheless, their joint study has not received attention so far, to the best of our knowledge. Our stylized model is the first attempt to jointly consider the effect of bid caps and disclosure policies.

The goal of our study of bid caps and disclosure policies is not to fulfill a mere theoretical curiosity; it is natural to believe that the same policy maker or legislative institution may have, to a varying extent, control over both the transparency requirements and the caps on expenditures of lobbyists. As both bid caps and disclosure policies build on the same key conflicting forces between symmetry/asymmetry among lobbyists, investigating how the interaction between these two plays out is appealing and far from trivial.

In building our stylized model, we borrow the most common and simple ingredients from the two above-mentioned strands of the literature. As typical in the strand of the

\footnotetext{
${ }^{1}$ This is no longer true when lobbyists' types are affiliated (Chen, 2019b).
} 
literature on bid caps, we model lobbying as an all-pay auction between two risk-neutral lobbyists seeking a political prize by choosing expenditures, and a cap on the lobbying expenditures applies to both lobbyists. As typically assumed for tractability in the strand of the literature on disclosure policies, lobbyists' valuation of the prize are drawn from a common binary distribution. ${ }^{2}$ A designer commits to disclose or conceal lobbyists' valuations before getting to know them, and may (or may not) condition the disclosure policy to the realization of valuations.

Concerning the bid cap, when looking separately at expenditures of low and high types, we find that the expenditures of low-types under full concealment or full-disclosure reach a maximum for some intermediate value of the bid cap. All other expenditures (i.e., those of low-types under partial disclosure policies, and those of high-types under any disclosure policy) increase in the bid cap. Nevertheless, when aggregating expenditures of low and high types, we find that the bid cap unambiguously decreases ex-ante total expenditures, regardless of the disclosure policy; that is, the non-monotonicity found in the expenditures of the low-type under full concealment is dominated by the increase of high-type expenditures. Hence, Che and Gale (1998) perverse effect of bid caps increasing expenditures is only present in our setup for low-types under full concealment and full disclosure, and it ex-ante disappears when considering the expected total expenditures. This is because in our setup bidders are ex-ante symmetric (though possibly ex-post asymmetric); hence, a bid cap that would have the Che and Gale (1998)'s effect of increasing expenditures when one player is strong and one weak is countered by the decrease in expenditures due to the bid cap in case both players are strong. Our results show that the latter effect always dominates the former, regardless of the disclosure policy. This is in line with the finding of Gavious et al. (2002) for linear costs and ex-ante symmetric players. While Gavious et al. (2002) consider a private information setup, we consider variable degrees of information; from complete information in case of full disclosure by the designer, to private information in case of full concealment by the designer, to intermediate levels of information if the designer commits to disclose only some specific realizations of types, and not others.

Concerning the disclosure policy, our main finding is that, for any given bid cap and allowing for full and partial disclosure policies, we find that expenditures are minimized with the policy in which a designer discloses bidders' types only if both players have a low valuation of the prize. The expenditures are maximized with the full concealment policy or with the disclosure in which a designer discloses the information only if both players have a high valuation. As our main motivating application is that of lobbying, where expenditures are typically seen as wasteful, we focus in what follows on the intuition

\footnotetext{
${ }^{2}$ Two exceptions are Morath and Münster (2008) and Fu et al. (2014), who consider a setup with continuum of types. However, in their setup there is no bid cap, and the designer has access only to full disclosure and full concealment policies, and not to partial disclosure policies.
} 
behind why expenditures are minimized if the designer commits to disclose valuations only if both players happen to have a low valuation of the prize. ${ }^{3}$ We denote this policy by $C C D$, where the first $C$ means disclosing when both players have a high valuation, the second $C$ means disclosing when one player has a high and one a low valuation, and the $D$ means disclosing when both players have a low valuation.

Intuition-CCD. A key change when considering partial information disclosure is that first- and higher-order beliefs potentially differ between players. In fact, under full concealment $C C C$, each type has the same belief over the rival's type (distributed according to the commonly known prior), and the same belief over the rival's belief over her own type (distributed according to the commonly known prior), and so on. Under partial information disclosure, the first-order beliefs may differ from the secondand higher-order beliefs. In fact, consider the expenditure-minimizing disclosure policy $C C D$. Then, low-types are always aware of their rival's valuation, ${ }^{4}$ high-types are always unaware of their rival's valuation, and the two previous facts are commonly known.

To see that first- and higher-order beliefs differ between players consider, for instance, the beliefs of a low-type when a high and a low compete:

1. first-order beliefs: the low knows her rival's type, and hence her beliefs are degenerate,

2. second-order beliefs: the low knows that her rival believes that the low herself could be high or low (according to the prior distribution of types).

Therefore, focusing on the effect of differences between first- and second-order beliefs, ${ }^{5}$ which, as mentioned above, are the key novelty when introducing partial disclosure policies, one can note two key effects of the expenditure-minimizing policy $C C D$ :

1. the presence of expenditure discouragement due to a low knowing of being up against a high who believes might be up against another high, and thus exerts considerable effort, which in turn discourages the effort of the low, and,

2. the absence of expenditure boost due to a high knowing of being up against a low who is not fully discouraged as she is not informed of being up against a high. ${ }^{6}$

\footnotetext{
${ }^{3}$ Nevertheless, other applications of our results entail an expenditure-maximizing designer, such as sports, contests for promotions among employees, and R\&D contests. When the goal is to minimize expenditure and the bid cap is endogenous, a 0 cap is trivially optimal.

${ }^{4}$ If a low-type observes concealment by the designer, she infers of being again a high type with probability 1 .

${ }^{5}$ Clearly, beliefs of order higher than second count too, but as there are two players and types are binary, those higher order beliefs parallel first- and second-order beliefs, and hence for simplicity we focus on first- and second-order beliefs.

${ }^{6}$ This expenditure boosting effect would be present, for instance, under a disclosure policy $D C C$, where the high-types are always informed and the low-types never informed of their rival's type.
} 
Hence, both forces behind the differences between first- and second-order beliefs point at expenditure reduction, thus making the policy $C C D$ the expenditure-minimizing disclosure policy.

Despite the expenditure-minimizing disclosure policy depending neither on the bid cap nor on the distribution of types (i.e., the ex ante probability of high and low types), the full ranking of disclosure policies does depend on both. In particular, we find that expenditures under $D D D$ are unambiguously lower than under $D C C$, but expenditures under $C C C$ could rank anywhere from inducing the highest expenditure to inducing the second-lowest expenditures, depending on the bid cap and on the distribution of types. We further explain in the main body.

Other related literature. On disclosure policies in contests, ${ }^{7}$ Zhang and Zhou (2016) applies Bayesian persuasion tools to the optimal disclosure in Tullock contests. However, they consider a setup where one player's type is common knowledge and the other's type could be disclosed or concealed. Here, we consider disclosure policies on both players' valuations. Serena (2019) considers full and partial disclosure policies in Tullock setups, where the distribution of players' types is binary. The intuition behind our results shares some common features with the one behind Serena (2019). Nevertheless, in Serena (2019) there is no bid cap and the contest is modeled as a Tullock contest, rather than an all-pay auction. Lu et al. (2018) extend Serena's work in an all-pay auction setup. $\mathrm{Fu}$ et al. (2014) consider the optimal disclosure policy in an all-pay auction setup with multiple prizes. Chen (2019a) considers affiliated types and finds that the selection of disclosure policy depends also on the number of potential participants. While all these works consider a binary distribution, none considers the presence of bid caps.

The present paper contributes to the literature on expenditure caps in two-player allpay auctions with incomplete information. Two-sided private information with continuum of types and ex-ante symmetric players is considered by Gavious et al. (2002). Sahuguet (2006) extends the analysis to ex-ante asymmetric players. Chen (2019b) considers the case of affiliated signals. Olszewski and Siegel (2019) consider large contests and exante asymmetric agents. Einy et al. (2016) consider one-sided private information and common valuation. Hence, our equilibrium characterization when the designer commits to full concealment contributes to this literature by adding the case of two-sided private information with ex-ante symmetric players and binary types. Nevertheless, our main contribution to the bid cap literature remains that of endogenizing the disclosure policy.

More formally, in Serena (2019)'s Tullock setup, the overall ranking of policies by expected total expenditure is either $D C C \geq D D D \geq C C C \geq C C D$ or $D C C \geq C C C \geq$ $D D D \geq C C D$. In Lu et al. (2018)'s all-pay auction setup, the overall ranking of policies

\footnotetext{
${ }^{7}$ We only discuss here the disclosure of contestants' types. Other information could be disclosed. For instance, Lim and Matros (2009), Fu et al. (2011), Fu et al. (2016), Feng and Lu (2016), Chen et al. (2017) and Chen et al. (2020) consider the strategic disclosure of the number of contestants.
} 
is $C C C \geq D C C \geq D D D \geq C C D$. Our ranking is identical to Lu et al. (2018)'s ranking for sufficiently high bid cap, hence confirming their findings, while for intermediate bid caps, the overall ranking we derive is one of the two found by Serena (2019) in a Tullock setup. This suggests that the introduction of a noise (moving from an all-pay auction to a Tullock contest) and the introduction of a bid cap generate mirroring effects in terms of resulting ranking of disclosure policies by expected total expenditure.

\section{Model}

Consider a contest with two potential risk neutral bidders, $i=1,2$, and one indivisible prize, normalized to 1 . The value of the prize is common to all potential bidders. Prior to bidding, each bidder learns her ability (or type) $t_{i} \in\{H, L\}, H=1>L>0$. A bidder has a probability $p \in(0,1)$ to be the $L$-type and $1-p$ to be the $H$-type. ${ }^{8}$ The type of a bidder is only observable to herself and the designer.

Each bidder $i$ faces an exogenously given bid cap $h>0$ and submits a bid $b_{i} \leq h$. Bids are submitted simultaneously and independently of each other. The bidder with the highest bid wins the prize, but all bidders pay their bids. Ties are resolved by random allocation with equal probabilities. Each bidder $i$ 's expected payoff is:

$$
W_{i}= \begin{cases}1-\frac{b_{i}}{t_{i}} & \text { if } b_{i}>b_{j} \\ -\frac{b_{i}}{t_{i}} & \text { if } b_{i}<b_{j} \\ \frac{1}{2}-\frac{b_{i}}{t_{i}} & \text { if } b_{i}=b_{j} .\end{cases}
$$

The designer maximizes the expected total revenue by publicly announcing and precommiting $^{9}$ to her disclosure policy $\mathcal{P}$ on bidders' types, before observing them; we denote by $E R^{\mathcal{P}}$ the expected total revenue under disclosure policy $\mathcal{P}$.

In Section 2, we consider a designer who can only commit to fully concealing $(\mathcal{P}=$ $C C C)$ or fully disclosing $(\mathcal{P}=D D D)$ the information about the types of all bidders that is, $\mathcal{P} \in\{C C C, D D D\}$, and we compare the expected revenue across the disclosure policies for a given triplet of parameters $(p, L, h) .{ }^{10}$ In Section 3, we consider a designer who has a stronger commitment power; he can commit to concealing or disclosing according to types' realizations. Such a stronger commitment power is easily implementable, for instance, in online contests. Formally, $\mathcal{P} \in\{C C C, D D D, D C C, C C D\}$ where the first (respectively, second and third) element is the concealment/disclosure which is imple-

\footnotetext{
${ }^{8}$ If $p=0$ or $p=1$ the information disclosure plays no role, and thus we neglect these cases from the beginning to avoid trivial case distinctions.

${ }^{9}$ It is beyond the scope of our paper to provide a thorough analysis on the issue of commitment.

${ }^{10}$ We take bid cap as given throughout the paper, and we comment in each section how a bid cap affects expenditures. Hence, our results have immediate implications on the model with endogenous bid cap and information policy.
} 
mented in case of realizations $\{H, H\}$ (respectively, $\{H, L\}$ and $\{L, L\}) .{ }^{11}$ We compare $E R^{C C C}, E R^{D D D}, E R^{D C C}$, and $E R^{C C D}$ for a given triplet of parameters $(p, L, h)$.

\section{Full Concealment versus Full Disclosure}

In this section, we first characterize the bidders' equilibrium behavior under full concealment (Subsection 2.1) and full disclosure (Subsection 2.2) and then compare the expected revenue across the two disclosure policies, varying the triplet $(p, L, h)$.

\subsection{Full Concealment}

We first consider the subgame in which the designer commits to policy $C C C$. In this case, the designer conceals the information on the types of the bidders, and thus bidders de facto play a private information all-pay auction. We characterize the unique symmetric equilibrium under policy $C C C$, which depends on the triplet $(p, L, h)$ in the following proposition.

Proposition 2.1 (Full Concealment). Under policy CCC, there is a unique symmetric equilibrium, which is given below.

1. Suppose $h \geq(1-p)+p L$. The L-type bids according to a uniform mixed strategy over the interval $[0, p L]$. The $H$-type bids according to a uniform mixed strategy over the interval $[p L,(1-p)+p L]$.

2. Suppose $h \in((1-p) / 2+p L,(1-p)+p L)$. The L-type bids according to a uniform mixed strategy over the interval $[0, p L]$. The $H$-type bids $h$ with a probability of $\frac{2[(1-p)+p L-h]}{1-p}$ and bids according to a uniform mixed strategy over the interval $[p L, 2 h-[(1-p)+p L]]$ with a probability of $\frac{2\left[h-\left(\frac{1-p}{2}+p L\right)\right]}{1-p}$.

3. Suppose $h \in[(1+p) L / 2,(1-p) / 2+p L]$. The L-type bids according to a uniform mixed strategy over the interval $[0, p L]$. The $H$-type bids $h$.

4. Suppose $h \in(L / 2,(1+p) L / 2)$. The $L$-type bids $h$ with a probability of $\frac{2[(1+p) L / 2-h]}{p L}$ and bids according to a uniform mixed strategy over the interval $[0,2 h-L]$ with a probability of $\frac{2(h-L / 2)}{p L}$. The $H$-type bids $h$.

5. Suppose $h \in(0, L / 2]$. Both the L-type and the H-type bid $h$.

\footnotetext{
${ }^{11}$ Note that all other policies (i.e., $D D C, D C D, C D D$, and $C D C$ ) are outcome-equivalent to $D D D$ as belief-updating is perfect; for instance, if realizations are $\{L, L\}$ are a low-type observes concealment under policy $D D C$, she knows that if her rival was a high-type, the principal would have disclosed types, and thus she infers that her rival must be a low-type. Hence, focusing on $\mathcal{P} \in\{C C C, D D D, D C C, C C D\}$ is without loss of generality.
} 
The ex ante expected revenue from each type of bidder $\left(E R_{L}^{C C C}\right.$ and $\left.E R_{H}^{C C C}\right)$ and the total expected revenue $\left(E R^{C C C}\right)$ can be calculated accordingly. For instance, when $h \in(L / 2,(1+p) L / 2)$, there are two bidders, each of whom has a probability of $p$ being the $L$-type, and hence, following from case 4 of the above proposition, the expected revenue from $L$-type bidders in this case is ${ }^{12}$

$$
\begin{aligned}
E R_{L}^{C C C}(h) & =\left[2 p^{2}+2 p(1-p)\right] \cdot\left[\frac{2\left(\frac{1+p}{2} L-h\right)}{p L} \cdot h+\frac{2\left(h-\frac{L}{2}\right)}{p L} \cdot \frac{2 h-L}{2}\right] \\
& =L-2(1-p) h,
\end{aligned}
$$

which is intuitively in line with Che and Gale (1998): a tighter cap (decreasing h) constrains the high and hence gives hope to the low, increasing $E R_{L}^{C C C}$. This parallelism is true even if their model is under complete information.

Corollary 2.1. Under policy $C C C$, the ex ante expected revenue of the designer is

$$
E R^{C C C}(h)= \begin{cases}2 h & \text { if } h \leq \frac{1}{2} L, \\ L & \text { if } h \in\left(\frac{1}{2} L, \frac{1+p}{2} L\right), \\ p^{2} L+2(1-p) h & \text { if } h \in\left[\frac{1+p}{2} L, \frac{1-p}{2}+p L\right), \\ p^{2} L+(1-p)^{2}+2 p(1-p) L & \text { if } h \geq \frac{1-p}{2}+p L .\end{cases}
$$

Precisely, the ex ante expected revenue from $L$-type bidders and that from $H$-type bidders, respectively, are

$$
E R_{L}^{C C C}(h)= \begin{cases}2 p h & \text { for } h<\frac{L}{2} \\ L-2(1-p) h & \text { for } h \in\left[\frac{L}{2}, \frac{1+p}{2} L\right) \\ p^{2} L & \text { for } h \geq \frac{1+p}{2} L\end{cases}
$$

and

$$
E R_{H}^{C C C}(h)= \begin{cases}2(1-p) h & \text { for } h<\frac{1-p}{2}+p L \\ (1-p)^{2}+2 p(1-p) L & \text { for } h \geq \frac{1-p}{2}+p L\end{cases}
$$

One key observation in the above proposition and corollary is that the ex ante expected bid of a bidder (as well as the expected revenue of the designer) is not affected by a bid cap unless the cap is below the threshold $(1-p) / 2+p L$. More specifically, a cap higher than $(1-p)+p L$ is not binding and does not affect bidders' equilibrium behavior at all, and this case boils down to the standard private information all-pay auction without cap. A cap in between $(1-p) / 2+p L$ and $(1-p)+p L$ will affect a $H$-type bidder's equilibrium

\footnotetext{
${ }^{12}$ We write $E R(h)$ omitting the dependency of $E R$ on $L$ and $p$. Wherever convenient, we also omit the dependency of $E R$ on $h$.
} 
behavior but does not affect her expected bid. The shift of mass from the mass point to the uniform distribution perfectly balances out, and this is why her expected bid does not change. A cap in between $(1+p) L / 2$ and $(1-p) / 2+p L$ will reduce the expected revenue from $H$-type bidders but still does not affect $L$-type bidders' strategic behavior. With a cap below $(1+p) L / 2$, the game becomes equivalent to an auction with complete information with both bidders being the $L$-type. Another key observation is that, though a bid cap in general reduces the expected bid, it has different effects on $L$-type and $H$ type bidders. It reduces $H$-type bidders' bids but can increase $L$-type bidders' ones. In fact, the expected revenue from $L$-type bidders achieves its maximum at $h=L / 2$.

\subsection{Full Disclosure}

We next consider the subgame in which the designer commits to policy $D D D$. In this case, the designer discloses the actual types of all bidders before they submit their bids, and bidders de facto play a complete information all-pay auction with a bid cap. The equilibrium is characterized by Proposition 1 of Che and Gale (1998).

Proposition 2.2 (Full Disclosure). Under policy DDD, in the subgame in which the realized pair of types is $\left(t_{i}, t_{j}\right)$, w.l.o.g., assuming $t_{i} \geq t_{j}$, there is a unique equilibrium.

1. If $h \leq \frac{t_{j}}{2}$, both bidders bid $h$.

2. If $h>\frac{t_{j}}{2}$, bidder $i$ bids according according to a uniform mixed strategy over the interval $\left[0, t_{j}\right]$; bidder $j$ bids 0 with a probability of $1-\frac{t_{j}}{t_{i}}$ and bids according to a uniform mixed strategy over the interval $\left[0, t_{j}\right]$ with a probability of $\frac{t_{j}}{t_{i}}$.

We use the above characterization to derive expected revenues in the following corollary.

Corollary 2.2. Under policy DDD, the ex ante expected revenue of the designer is

$$
E R^{D D D}(h)= \begin{cases}2 h & \text { if } h \leq \frac{L}{2}, \\ p^{2} \cdot L+(1-p)^{2} \cdot 2 h+2 p(1-p) \cdot \frac{L}{2}(1+L) & \text { if } h \in\left(\frac{L}{2}, \frac{1}{2}\right) \\ p^{2} \cdot L+(1-p)^{2}+2 p(1-p) \cdot \frac{L}{2}(1+L) & \text { if } h \geq \frac{1}{2} .\end{cases}
$$

Precisely, the ex ante expected revenue from $L$-type bidders and that from $H$-type bidders, respectively, are

$$
E R_{L}^{D D D}(h)= \begin{cases}2 p h & \text { for } h \leq \frac{L}{2} \\ p^{2} L+2(1-p) p \cdot \frac{L}{2} \cdot L & \text { for } h>\frac{L}{2}\end{cases}
$$

and 


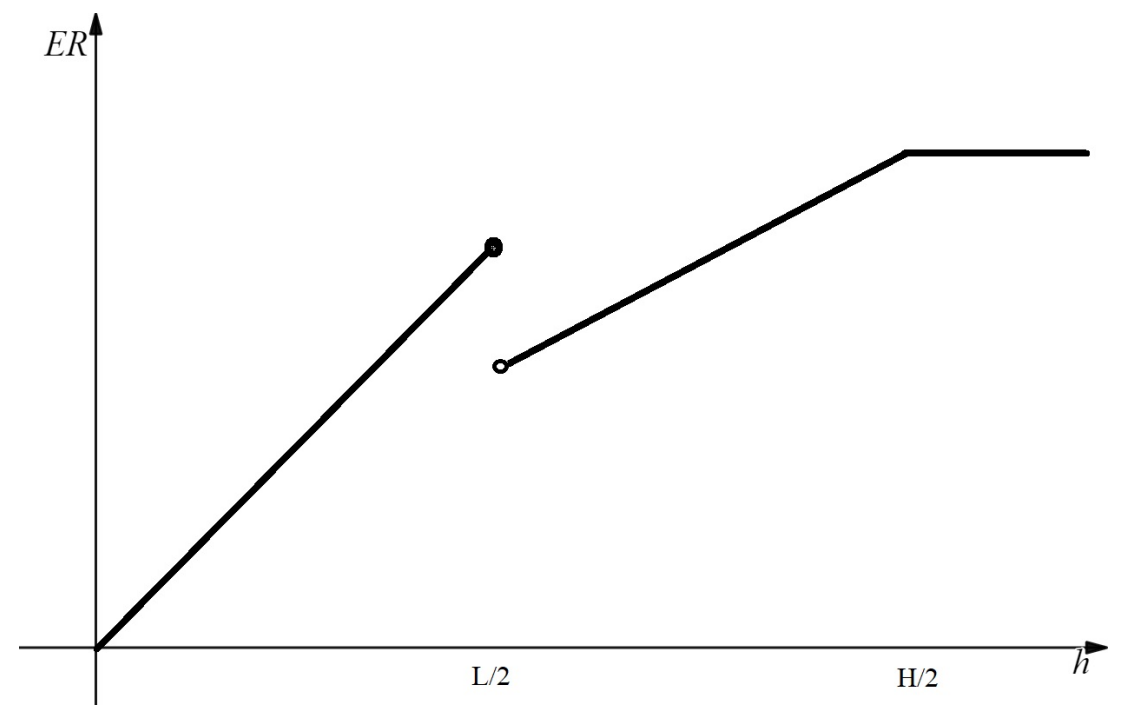

Figure 1: Expected revenue under full disclosure

$$
E R_{H}^{D D D}(h)= \begin{cases}2(1-p) h & \text { for } h \leq \frac{L}{2} \\ 2(1-p) p \cdot \frac{L}{2}+2(1-p)^{2} h & \text { for } h \in\left(\frac{L}{2}, \frac{1}{2}\right) \\ 2(1-p) p \cdot \frac{L}{2}+(1-p)^{2} & \text { for } h \geq \frac{1}{2} .\end{cases}
$$

Under full disclosure, a cap would affect the expected payment of a bidder (as well as the expected revenue of the designer) if and only if it is below the threshold $1 / 2$. More specifically, a cap in between $L / 2$ and $1 / 2$ will only affect the expected revenue in the case in which both bidders' realized types are $H$. A cap below $L / 2$ will affect the expected revenue in all cases of realized pairs of types.

Che and Gale (1998) consider the case in which the realized pair of types is $(H, L)$. They find that the expected revenue is discontinuous at $h=L / 2$. Figure 1 illustrates the ex ante expected revenue in corollary 2.2. The discontinuity at $L / 2$ is the same as that of Che and Gale (1998) (see Figure 1 in Che and Gale, 1998), which boils down to our setup when only considering realization pair $(H, L)$. After $L / 2$, in Che and Gale's setup, the revenue is constant for changes in the cap. However, in our setting, considering also realizations $(L, L)$ and $(H, H)$ makes the revenue increasing when the cap is above $L / 2$. Moreover, the revenue may not achieve its maximum at $h=L / 2$. It achieves its maximum at this point if and only if bidders have large probabilities of being the $L$-type (high $p$ ) and the $H$-type does not differ from the low-type too much ( $L$ close to 1 ).

\subsection{Full Concealment, Full Disclosure and Revenue Ranking}

Now, we are ready to compare the expected revenue across the two disclosure policies. Let us start with a special case in which there is no bid cap and $L$ approaches 0 , so that de facto the number of participating bidders could be 0,1 , or 2 . It is equivalent to a standard all-pay auction with a stochastic number of participating bidders (Chen et al., 2020). As 
found by Lim and Matros (2009), as well as Fu et al. (2011) and Chen et al. (2017), information disclosure is irrelevant to the expected revenue. This is because disclosing the number of participating bidders does not create any asymmetry among participating (or active) bidders, who, if any, are high types. ${ }^{13}$ However, Chen et al. (2020) find that, in this case, as long as there is a non-trivial bid cap, disclosure leads to a lower expected revenue. This is because under full disclosure a bidder's bidding strategy varies across the realized number of participating bidders. A bid cap limits her bids, and then there is no high bids to mitigate low bids any more.

Now, come back to the case in which $L$ is not arbitrarily close to 0 . In contrast to the case with stochastic entry, in this case, revenue comes from both types of bidders. Thus, we need to analyze the effects of information disclosure on both types of bidders. Similar to the case with stochastic entry, $H$-type bidders would bid in expectation more aggressively under full concealment, but this is not because of a bid cap. $L$-type bidders, however, could in expectation bid more aggressively under full disclosure. Formally, we state these properties along with others below.

Property 2.1. Consider $h>L / 2$.

1. Compared to full concealment, full disclosure causes revenue loss from $H$-type bidders, and the loss is increasing in the bid cap (i.e., $E R_{H}^{D D D}(h)-E R_{h}^{C C C}(h)$ is strictly negative and decreasing in $h$ ).

2. Compared to full concealment, full disclosure causes revenue loss from L-type bidders if and only if the bid cap is below $\frac{L}{2}[1+p(1-L)]$, and the revenue loss is decreasing and the revenue gain is increasing in the bid cap. In particular,

$$
E R_{L}^{D D D}(h)-E R_{L}^{C C C}(h)\left\{\begin{array}{l}
=0 \text { for } h \leq \frac{L}{2} \text { or } h=\frac{L}{2}[1+p(1-L)] \\
<0 \text { for } h \in\left(\frac{L}{2}, \frac{L}{2}[1+p(1-L)]\right. \\
>0 \text { for } h>\frac{L}{2}[1+p(1-L)]
\end{array}\right.
$$

and increases in $h$.

3. Compared to no bid cap, a bid cap causes revenue loss from $H$-type bidders under both disclosure policies, has no effect on revenue from L-type bidders under full disclosure, but increases revenue from L-type bidders under full concealment.

4. Under full concealment, the H-type's and the L-type's bids are perfect strategic substitutes for $h \in\left(\frac{L}{2}, \frac{L}{2}[1+p(1-L)]\right)$, in the sense that the expected total bid remains constant as the bid cap changes in this interval.

\footnotetext{
${ }^{13}$ Compared to full concealment, the drawback of full disclosure is that a participating bidder will bid 0 when facing no competitor, and the benefit is that a participating bidder will exert maximal effort when facing another competitor. The two effects perfectly balance out.
} 
In particular, in the absence of a (binding) cap, information disclosure has the opposite effects on $H$-type bidders and $L$-type bidders. While $H$-type bidders will in expectation bid more under full concealment than under full disclosure, $L$-type bidders will bid more under full disclosure. Under full concealment, $L$-type bidders would fear to bid too much because there is a chance that her opponent is of $H$-type. Under full disclosure, there is a chance that a $H$-type bidder faces a $L$-type bidder, in which case the $H$-type bidder does not need to bid high to win (Lazear and Rosen, 1981). However, the difference in the expected revenue from $H$-type bidders dominates, and this leads to a higher expected revenue under full concealment (Morath and Münster, 2008; Fu et al., 2014; Lu et al., 2018). Now, a bid cap has different effects on $H$-type and $L$-type bidders, and thus it can change the revenue ranking of disclosure policies. Let

$$
\bar{x}:=\frac{L}{2}(1+K)-\frac{1+L}{2}(K-p)(L-K) .
$$

The main finding is stated below.

Theorem 2.1 (Revenue Ranking). Denote $K=\min \left\{L, \frac{1}{1+L}\right\}$. If $p \geq K$, then

$$
E R^{D D D}(h)-E R^{C C C}(h) \begin{cases}<0 & \text { if } h>\frac{L}{2} \\ =0 & \text { if } h \leq \frac{L}{2}\end{cases}
$$

If $p<K$, then

$$
E R^{D D D}(h)-E R^{C C C}(h) \begin{cases}>0 & \text { if } h \in\left(\frac{L}{2} \cdot \frac{1-p L}{1-p}, \bar{x}\right) \\ <0 & \text { if } h \in\left(\frac{L}{2}, \frac{L}{2} \cdot \frac{1-p L}{1-p}\right) \cup(\bar{x},+\infty) \\ =0 & \text { if } h \leq \frac{L}{2} \text { or } h=\bar{x} .\end{cases}
$$

The key insight from the above theorem is that the classic intuition developed in Lazear and Rosen (1981), in which leveling the playing field of the competitors leads to revenue maximization, does not extend to the scenario in which competitors face bid constraints. Here, a designer may want to create asymmetry between bidders. This is due to the difference in the effects of a bid cap and the disclosure policies across different types of bidders. There can be two counter effects: $H$-type bidders bid more aggressively in expectation under full concealment than under full disclosure, while $L$-type bidders might shirk under full concealment. Due to property 2.1, under an intermediate level of bid cap, the revenue gain from $L$-type bidders caused by information disclosure could outweigh the revenue loss from $H$-type bidders. Thus, full disclosure becomes more effective in raising revenue in this case. The following example illustrates the two cases of the above theorem.

\section{Example 2.1.}


a. $L=0.5$ and $p=4 / 5$.

b. $L=0.5$ and $p=2 / 5$.

In Example 2.1.a, each bidder has a large chance of being the $L$-type. Figure 2 shows how the expected revenue from each type of bidder changes in the bid cap. The revenue loss from $H$-type bidders caused by information disclosure outweighs the revenue gain from $L$-type bidders, if any, and thus full concealment dominates full disclosure. More specifically, the bid cap has little effect on $H$-type bidders under full disclosure (the $E R_{H}^{D D D}$ curve is almost flat when $h>L / 2$ ), and thus the expected total revenue under full disclosure is below $L$ due to the Che-and-Gale drop of the expected revenue from $L$-type bidders when the bid cap increases from just below $L / 2$ to just above $L / 2$. The expected revenue under full concealment is, however, at least $L$ due to the substitutiveness of the $H$-type's and the $L$-type's efforts.

By contrast, in example 2.1.b, each bidder has a small chance of being the $L$-type. It is illustrated by Figure 3. The bid cap has strong effects on $H$-type bidders under both disclosure policies. This creates the intermediate range of the bid cap $(1 / 3,3 / 8)$, in which the revenue gain from L-type bidders caused by information disclosure can outweigh the revenue loss from $H$-type bidders and thus full concealment no longer dominates full disclosure.

To examine it in detail, let us raise the bid cap from 0 .

- When $h \in(0, L / 2]$, a low bid cap makes type realizations irrelevant, and thus the expected revenue remains the same across disclosure policies.

- When $h$ just passes $L / 2$, full concealment suddenly becomes superior because: (1) $E R_{H}^{C C C}, E R_{L}^{C C C}$, and $E R_{H}^{D D D}$ all change smoothly in $h ;(2)$ however, $E R_{L}^{D D D}$ displays the Che-and-Gale drop at $h=L / 2$, above which the $L$-type loses some hope to win.

- When $h>L / 2$, the bid cap has different effects on $L$-type and $H$-type bidders across the disclosure policies. On the one hand, considering the $L$-type, (1) $E R_{L}^{D D D}$ is constant, (2) $E R_{L}^{C C C}$ is strictly decreasing in $h$ until a certain point, and (3) $E R_{L}^{C C C}>E R_{L}^{D D D}$ when $h$ is large (the higher $h$ is, the more $L$-type bidders will shirk under full concealment). On the other hand, considering the $H$-type, (i) $E R_{H}^{C C C}$ and $E R_{H}^{D D D}$ are both increasing in $h$; (ii) $E R_{H}^{C C C}=E R_{H}^{D D D}$ when $h=$ $L / 2$; (iii) however, $E R_{H}^{C C C}$ increases faster than $E R_{H}^{D D D}$ in $h$. This creates the intermediate range of $h,(1 / 3,3 / 8)$, in which $E R_{L}^{D D D}-E R_{L}^{C C C}>E R_{H}^{C C C}-E R_{H}^{D D D}$. That is, the bid cap is high enough for $L$-type bidders to shirk a lot under full concealment but low enough to prevent $H$-type bidders to bid too aggressively under full concealment. When $h$ is above $3 / 8, E R_{H}^{C C C}-E R_{H}^{D D D}$ dominates $E R_{L}^{D D D}-$ $E R_{L}^{C C C}$. 


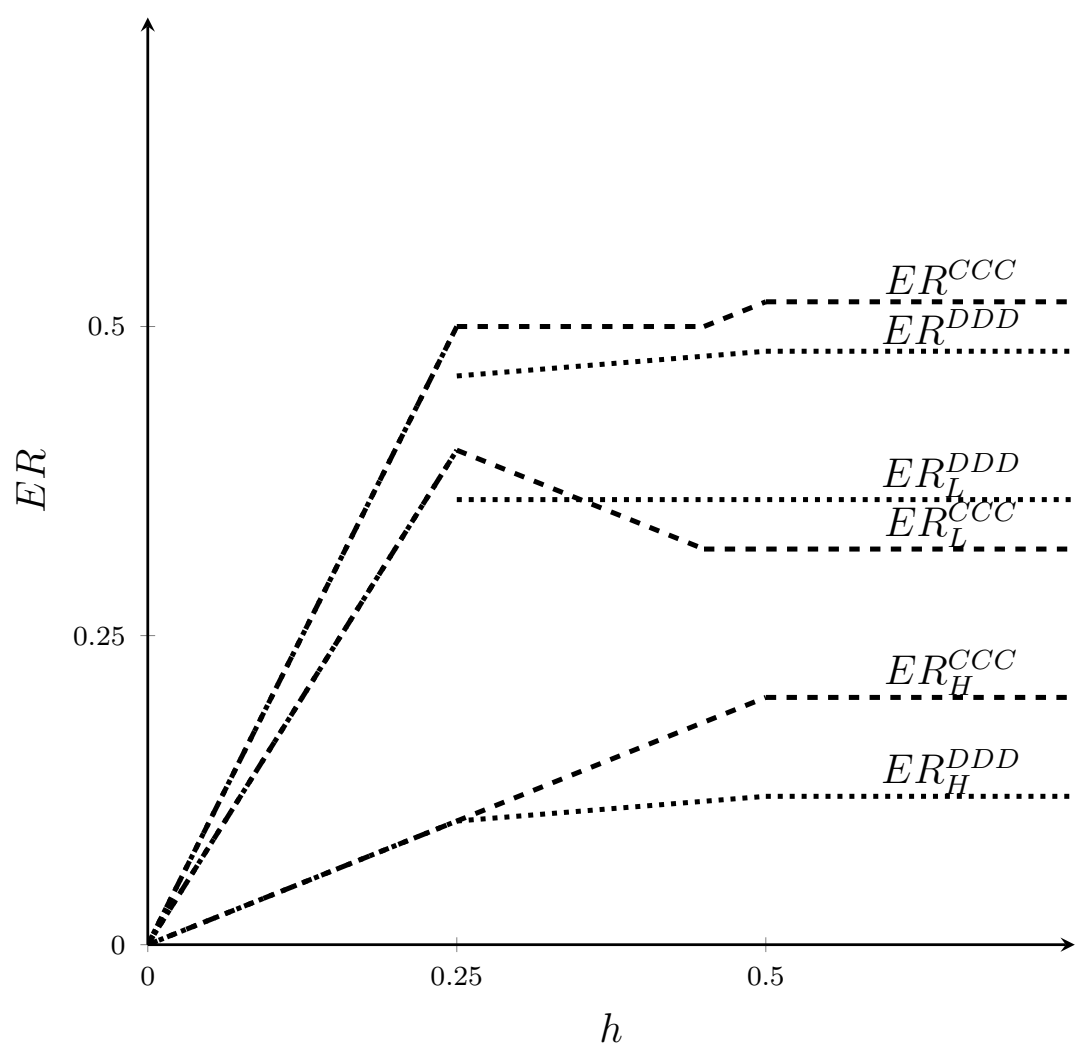

Figure 2: This is for Example 2.1.a in which $L=0.5$ and $p=4 / 5$.

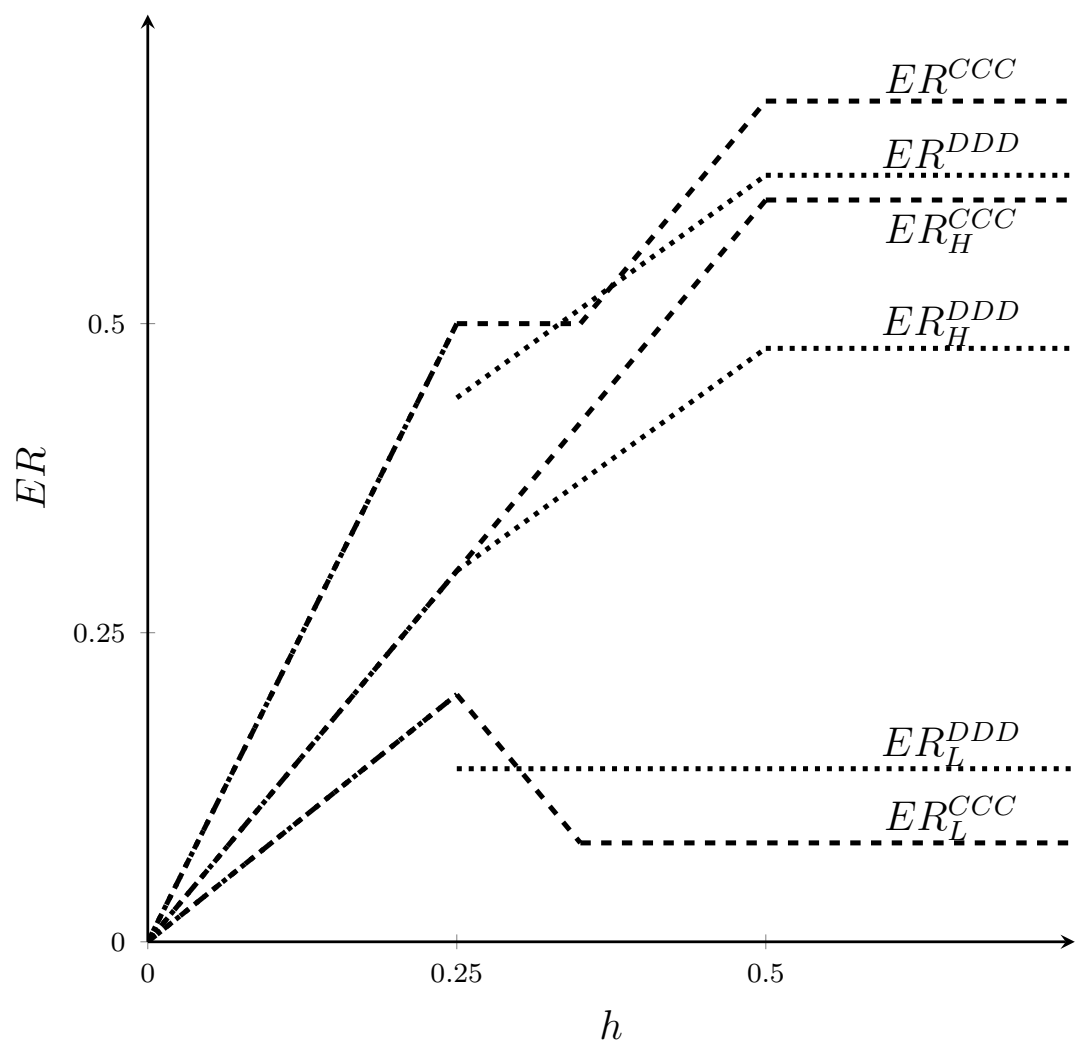

Figure 3: This is for Example 2.1.b in which $L=0.5$ and $p=2 / 5$. 


\section{Partial Disclosure and Revenue Ranking}

So far, the designer could only commit to fully concealing or fully disclosing types. However, there are real-life examples, especially in online contests, where the principal has a stronger commitment power. In this section we ask if the principal can do any better than fully concealing or fully disclosing by using a partial disclosure policy. The answer is often positive, within the family of partial disclosure policies where the principal commits to concealing or disclosing according to the realization of types; that is, $\mathcal{P} \in\{C C C, D D D, D C C, C C D\}$. In the following, we characterize the unique symmetric equilibrium under each of the partial disclosure policy $(C C D$ and $D C C)$ and then compare the expected revenue across all disclosure policies.

\subsection{Partial Disclosure Policy- $D C C$}

In the following proposition we characterize the equilibrium when $C$ occurs under policy $D C C$. Note that this implies that the $H$-type (if any) is aware of the rival's type, the $L$-type is not aware of the rival's type, and these two facts are common knowledge. Let

$$
\underline{x}:=\frac{p(1-L) L}{1-p L} .
$$

Then, we obtain the following.

Proposition 3.1. For partial disclosure policy DCC, the following is the unique symmetric equilibrium when $C$ occurs.

1. Suppose $h \geq L$. The L-type bids according to a uniform mixed strategy over the interval $[0, \underline{x}]$ with a probability of $\frac{\underline{x}}{p L}$ and bids according to a uniform mixed strategy over the interval $[\underline{x}, L]$ with a probability of $1-\frac{\underline{x}}{p L}$. The $H$-type bids according to a uniform mixed strategy over the interval $[\underline{x}, L]$.

2. Suppose $h \in[(\underline{x}+L) / 2, L)$. The L-type bids $h$ with a probability of $2(L-h)$ and bids according to a uniform mixed strategy over the interval $[0, \underline{x}]$ with a probability of $\underline{\underline{x}}$ and bids according to a uniform mixed strategy over the interval $[\underline{x}, 2 h-L]$ with a probability of $2[h-(L+\underline{x}) / 2]$. The $H$-type bids $h$ with a probability of $\frac{2(1-p L)(L-h)}{(1-p) L}$ and bids according to a uniform mixed strategy over the interval $[\underline{x}, 2 h-L]$ with a probability of $\frac{(1-p L)[h-(\underline{x}+L) / 2]}{(1-p) L}$.

3. Suppose $h \in(L / 2,(\underline{x}+L) / 2)$. The L-type bids $h$ with a probability of $\frac{2[(1+p) L / 2-h]}{p L}$ and bids according to a uniform mixed strategy over the interval $[0,2 h-L]$ with a probability of $\frac{2(h-L / 2)}{p L}$. The $H$-type bids $h$.

4. Suppose $h \leq L / 2$. Both the L-type and the H-type bid $h$. 
Note that, under disclosure policy $D C C$, when $D$ occurs the equilibrium is the same as that characterized in Proposition 2.2 with $t_{1}=t_{2}=H$. Propositions 2.2 and 3.1 together yield the ex ante expected revenue under policy $D C C$.

Corollary 3.1. Under policy DCC, the ex ante expected revenue is stated below.

1. Suppose $p \geq \frac{1}{2 L}$, then

$$
E R^{D C C}(h)= \begin{cases}2 h & \text { for } h \leq \frac{L}{2} \\ L & \text { for } h \in\left(\frac{L}{2}, \frac{1}{2}\right) \\ (1-p)^{2}(1-2 h)+L & \text { for } h \in\left[\frac{1}{2}, \underline{x}\right) \\ (1-p)^{2}(1-2 \underline{x})+L & \text { for } h \geq \underline{x} .\end{cases}
$$

2. Suppose $p<\frac{1}{2 L}$, then

$$
E R^{D C C}(h)= \begin{cases}2 h & \text { for } h \leq \frac{L}{2} \\ L & \text { for } h \in\left(\frac{L}{2}, \underline{x}\right) \\ (1-p)^{2}(2 h-2 \underline{x})+L & \text { for } h \in\left[\underline{x}, \frac{1}{2}\right) \\ (1-p)^{2}(1-2 \underline{x})+L & \text { for } h \geq \frac{1}{2} .\end{cases}
$$

Note that when $p \geq \frac{1}{2 L}$, a bid cap can increase the expected revenue under policy $D C C$. Any cap $h \in(L / 2,1 / 2)$ is optimal. In fact, a designer maximizing revenue finds it optimal to have a bid cap $h \in(L / 2,1 / 2)$ (corollary 3.1). This is because under $D C C$, the subgame that follows the realization of $C$ is an auction with correlated types. As noted by Chen (2019b), a bid cap in an auction with correlated types can increase expected revenue. However, we will show that the positive effect of a bid cap is never the dominant one when the disclosure policy is endogenous; when the designer can choose both information disclosure policy and bid cap, it is optimal to employ full concealment with no bid cap.

\subsection{Partial Disclosure Policy- $C C D$}

In the following proposition we characterize the equilibrium when $C$ occurs under policy $C C D$. Note that this implies that the $L$-type (if any) is aware of the rival's type, the $H$-type is not aware of the rival's type, and these two facts are common knowledge.

Proposition 3.2. For partial disclosure policy CCD, the following is the unique symmetric equilibrium when $C$ occurs.

1. Suppose $L \leq 1-p$. 
(a) Suppose $h \geq 1-p$. The L-type bids 0 . The $H$-type bids according to a uniform mixed strategy over the interval $[0,1-p]$.

(b) Suppose $h \in((1-p) / 2,1-p)$. The L-type bids 0 . The $H$-type bids $h$ with a probability of $\frac{2[(1-p)-h]}{1-p}$ and bids according to a uniform mixed strategy over the interval $[0,2 h-(1-p)]$ with a probability of $\frac{2[h-(1-p) / 2]}{1-p}$.

(c) Suppose $h \in(L / 2,(1-p) / 2]$. The L-type bids 0 . The H-type bids $h$.

2. Suppose $L>1-p$.

(a) Suppose $h \geq L$. The L-type bids 0 with a probability of $\frac{1-L}{p}$ and bids according to a uniform mixed strategy over the interval $[0, L]$ with a probability of $\frac{L-(1-p)}{p}$. The H-type bids according to a uniform mixed strategy over the interval $[0, L]$.

(b) Suppose $h \in(L / 2, L)$. The L-type bids 0 with a probability of $\frac{1-L}{p}$, bids $h$ with a probability of $\frac{2(L-h)[L-(1-p)]}{p L}$, and bids according to a uniform mixed strategy over the interval $[0,2 h-L]$ with a probability of $\frac{2(h-L / 2)[L-(1-p)]}{p L}$. The $H$ type bids $h$ with a probability of $\frac{2(L-h)}{L}$ and bids according to a uniform mixed strategy over the interval $[0,2 h-L]$ with a probability of $\frac{2(h-L / 2)}{L}$.

3. Suppose $h \leq L / 2$. Both the L-type and the H-type bid $h$.

Propositions 2.2 and 3.2 together yield the ex ante expected revenue under policy $C C D$.

Corollary 3.2. Under policy CCD, the ex ante expected revenue is given below.

1. Suppose $L>1-p$, then

$$
E R^{C C D}(h)= \begin{cases}2 h & \text { for } h \leq \frac{L}{2} \\ {[p+(1-p) L] L} & \text { for } h>\frac{L}{2}\end{cases}
$$

2. Suppose $L \leq 1-p$, then

$$
E R^{C C D}(h)= \begin{cases}2 h & \text { for } h \leq \frac{L}{2} \\ p^{2} L+2(1-p) h & \text { for } h \in\left(\frac{L}{2}, \frac{1-p}{2}\right) \\ p^{2} L+(1-p)^{2} & \text { for } h \geq \frac{1-p}{2}\end{cases}
$$

\subsection{Ranking Disclosure Policies}

In what follows we omit the dependence of $E R$ on $h$ for the sake of space. We first derive some partial results in the following lemma. 
Lemma 3.1. Suppose $h \leq L / 2$, then $E R^{D C C}=E R^{C C C}=E R^{D D D}=E R^{C C D}$. Suppose $h>L / 2$.
i. $E R^{C C C}>E R^{C C D}$.
ii. $E R^{D C C}>E R^{D D D}>E R^{C C D}$.
iii. If $p \geq \frac{1}{2 L}, E R^{C C C}>E R^{D C C}$.

Proof. For part iii, note that $\frac{(1+p) L}{2}>\frac{L}{2}\left(\frac{p(1-L)}{1-p L}+1\right)$. Thus, $E R^{D C C}<L=E R^{C C C}$ for $L>\frac{1}{2}$. For the other parts, see the appendix.

From the above lemma, we observe: (1) policy $C C D$ always leads to the lowest effort provision, since this policy is not effective in eliciting effort from the $L$-type (while the $H$-type is capped); (2) policy $D D D$ is always dominated by policy $D C C$. From the above Lemma we know that the revenue-maximizing policy is either $D C C$ or $C C C$. To have a full ranking on the disclosure policies, we need to identify the conditions for $E R^{C C C}>E R^{D C C}, E R^{C C C} \in\left[E R^{D C C}, E R^{D D D}\right]$, and $E R^{C C C} \in\left[E R^{D D D}, E R^{C C D}\right]$. The main result is stated below.

Theorem 3.1. Recall that $K:=\min \left\{L, \frac{1}{1+L}\right\}$ Generically, there are five possible rankings, depending on the triplet $(p, L, h)$.

$i$. Suppose $p \geq \frac{1}{2 L}$, the domain of $h$ can be divided into three regions

ii. Suppose $p \in\left[K, \frac{1}{2 L}\right)$, the domain of $h$ can be divided into four regions. ${ }^{14}$

iii. Suppose $p<K$, the domain of $h$ can be divided into seven regions.

The partition of each case and the revenue ranking in each region of each case are in the table below.

\begin{tabular}{|c|c|c|c|}
\hline \multirow{2}{*}{ Ranking } & \multicolumn{3}{|c|}{ Regions of $h$} \\
\cline { 2 - 4 } & If $p \geq \frac{1}{2 L}$ & If $p \in\left[K, \frac{1}{2 L}\right)$ & If $p \in(0, K)$ \\
\hline$E R^{D C C}=E R^{C C C}=E R^{D D D}=E R^{C C D}$ & $\left(0, \frac{L}{2}\right]$ & $\left(0, \frac{L}{2}\right]$ & $\left(0, \frac{L}{2}\right]$ \\
\hline$E R^{D C C}=E R^{C C C}>E R^{D D D}>E R^{C C D}$ & $\left(\frac{L}{2}, \frac{1}{2}\right)$ & $\left(\frac{L}{2}, \underline{x}\right)$ & $\left(\frac{L}{2}, \underline{x}\right)$ \\
\hline$E R^{D C C}>E R^{C C C}>E R^{D D D}>E R^{C C D}$ & $\backslash$ & $(\underline{x}, \hat{h})$ & $\left(\underline{x}, \frac{L}{2} \cdot \frac{1-p L}{1-p}\right) \cup(\bar{x}, \tilde{h})$ \\
\hline$E R^{D C C}>E R^{D D D}>E R^{C C C}>E R^{C C D}$ & $\backslash$ & $\backslash$ & $\left(\frac{L}{2} \cdot \frac{1-p L}{1-p}, \bar{x}\right)$ \\
\hline$E R^{C C C}>E R^{D C C}>E R^{D D D}>E R^{C C D}$ & $\left(\frac{1}{2},+\infty\right)$ & $(\hat{h},+\infty)$ & $(\tilde{h},+\infty)$ \\
\hline
\end{tabular}

The $\hat{h}$ value in case ii is in between $\underline{x}$ and $\frac{1-p}{2}+p L$ and the $\tilde{h}$ value in case iii is in between $\bar{x}$ and $\frac{1-p}{2}+p L$.

\footnotetext{
${ }^{14}$ Note that $\frac{1}{2 L}>\frac{1}{1+L} \geq K$.
} 
Proof. Case $i$ follows from Lemmas 3.1 immediately. Case $i i$ follows Lemma 3.1.ii and a direct comparison between $E R^{D C C}$ and $E R^{C C C}$ for $h \in\left[\underline{x}, \frac{1-p}{2}+p L\right]$. Case iii follows from Lemma 3.1.ii, Theorem 2.1, and a direct comparison between $E R^{D C C}$ and $E R^{C C C}$ for $h \in\left[\bar{x}, \frac{1-p}{2}+p L\right]$.

Hence, all in all, we find that, for any given bid cap, ex ante expected expenditures are minimized by the policy in which the designer commits to disclosing valuations only if both players happen to have a low valuation of the prize (policy $C C D$ ). Despite our focus being on expenditure minimization, as our main application is that of lobbying, Theorem 3.1 characterizes the full ranking of disclosure policies, including the expenditure maximizing one, which is either to fully concealing types or to disclose valuations only if both players happen to have a high valuation of the prize. This is in line with Serena (2019) and $\mathrm{Lu}$ et al. (2018). More specifically, our ranking is identical to Lu et al. (2018)'s ranking for sufficiently high bid cap, hence confirming their findings, while for intermediate bid caps, the overall ranking we derive is one of the two found by Serena (2019) in a Tullock setup. This suggests that the introduction of a noise (moving from an all-pay auction to a Tullock contest) and the introduction of a bid cap generate mirroring effects in terms of resulting ranking of disclosure policies by expected total expenditure.

\section{Conclusion}

In this paper, we examined the effect of disclosing the actual types of bidders in an all-pay auction with a common exogenous bid cap. Despite deriving some interim nonmonotonic effects of the bid cap on expenditures (for low-types under full concealment or full-disclosure and for partial disclosure policies), the bid cap unambiguously decreases the expected total expenditures, regardless of the disclosure policy. For any given bid cap and allowing for full and partial disclosure policies, we find that expenditures are minimized with the policy in which a designer discloses bidders' types only if both players have a low valuation of the prize. The expenditures are maximized with the full concealment policy or with the disclosure in which a designer discloses the information only if both players have a high valuation.

\section{References}

Che, Y. and I.L. Gale (1998) "Caps on political lobbying," American Economic Review, Vol. 88, No. 3, pp. 643-651.

Chen, B. (2019a) "Disclosure policies in all-pay auctions with affiliated abilities," Available at SSRN 3406595. 
- (2019b) "On the effects of bid caps in all-pay auctions," Economics Letters, Vol. 177, pp. 60-65.

Chen, B., X. Jiang, and D. Knyazev (2017) "On disclosure policies in all-pay auctions with stochastic entry," Journal of Mathematical Economics, Vol. 70, pp. 66-73.

Chen, B., L. Ma, Z. Zhu, and Y. Zhou (2020) "Disclosure policies in all-pay auctions with bid caps and stochastic entry," Economics Letters, Vol. 186, p. 108805.

Einy, E., O. Haimanko, R. Orzach, and A. Sela (2016) "Common-value all-pay auctions with asymmetric information and bid caps," International Journal of Game Theory, Vol. 45, No. 1-2, pp. 63-88.

Feng, X. and J. Lu (2016) "The optimal disclosure policy in contests with stochastic entry: A Bayesian persuasion perspective," Economics Letters, Vol. 147, pp. 103-107.

Fu, Q., Q. Jiao, and J. Lu (2011) "On disclosure policy in contests with stochastic entry," Public Choice, Vol. 148, No. 3-4, pp. 419-434.

- (2014) "Disclosure policy in a multi-prize all-pay auction with stochastic abilities," Economics Letters, Vol. 125, No. 3, pp. 376-380.

Fu, Q., J. Lu, and J. Zhang (2016) "Disclosure policy in Tullock contests with asymmetric stochastic entry," Canadian Journal of Economics/Revue canadienne d'économique, Vol. 49, No. 1, pp. 52-75.

Gavious, A., B. Moldovanu, and A. Sela (2002) "Bid costs and endogenous bid caps," RAND Journal of Economics, pp. 709-722.

Lazear, E.P. and S. Rosen (1981) "Rank-order tournaments as optimum labor contracts," Journal of political Economy, Vol. 89, No. 5, pp. 841-864.

Lim, W. and A. Matros (2009) "Contests with a stochastic number of players," Games and Economic Behavior, Vol. 67, No. 2, pp. 584-597.

Lu, J., H. Ma, and Z. Wang (2018) "Ranking disclosure policies in all-pay auctions," Economic Inquiry, Vol. 56, No. 3, pp. 1464-1485.

Morath, F. and J. Münster (2008) "Private versus complete information in auctions," Economics Letters, Vol. 101, No. 3, pp. 214-216.

Olszewski, W. and R. Siegel (2019) "Bid caps in large contests," Games and Economic Behavior, Vol. 115, pp. 101-112.

Sahuguet, N. (2006) "Caps in asymmetric all-pay auctions with incomplete information," Economics Bulletin, Vol. 3, No. 9, pp. 1-8. 
Serena, M. (2019) "Harnessing beliefs to stimulate efforts," Available at SSRN 2686543.

Szech, N. (2015) "Tie-breaks and bid-caps in all-pay auctions," Games and Economic Behavior, Vol. 92, pp. 138-149.

Zhang, J. and J. Zhou (2016) "Information disclosure in contests: A Bayesian persuasion approach," Economic Journal, Vol. 126, No. 597, pp. 2197-2217.

\section{Appendix}

\section{Appendix A: Symmetric equilibrium under policy $C C C$}

Let $F_{L}(\cdot)$ and $F_{H}(\cdot)$ be the $L$-type's and the $H$-types's distributions of bids, respectively, in a symmetric equilibrium. Let $G(\cdot): p F_{L}(\cdot)+(1-p) F_{H}(\cdot)$ be a bidder's (ex ante) distribution of bids in symmetric equilibrium. Let $\bar{b}_{L} \equiv \inf \left\{z \mid F_{L}(z)=1\right\}$ and $\underline{b}_{H} \equiv$ $\inf \left\{z \mid F_{H}(z)>0\right\}$ be the $L$-type's uperimum bid and the $H$-type's infimum bid, respective.

Lemma 4.1. In a (symmetric) equilibrium bidding strategy, there is no mass point at any bid $b \in[0, h)$.

Proof. For any bid $b \in(0, h)$, see Che and Gale (1998) for the case with complete information. For $b=0$, suppose $G(0)>0$, then a bidder who has mass at 0 can profitably move weight from 0 to slightly above 0 .

\section{Lemma 4.2.}

$$
\begin{aligned}
i \bar{b}_{L} & \leq \underline{b}_{H} ; \\
i i \bar{b}_{L} & =\underline{b}_{H}, \text { if } \underline{b}_{H}<h .
\end{aligned}
$$

Proof. [i]. Suppose $\bar{b}_{L}>\underline{b}_{H}$. Then there is a non-empty interval $I \subset\left[\operatorname{supp}\left(F_{H}\right) \cap \operatorname{supp}\left(F_{L}\right)\right]$ such that both the $L$-type and the $H$-type are indifferent between any two bids $x$ and $x^{\prime}$ in $I$. That is

$$
E U_{v_{i}}(x)-E U_{v_{i}}\left(x^{\prime}\right)=0 \Rightarrow\left[G(x)-\frac{x}{v_{i}}\right]-\left[G\left(x^{\prime}\right)-\frac{x^{\prime}}{v_{i}}\right]=0 \Rightarrow\left[G(x)-G\left(x^{\prime}\right)\right] v_{i}=x-x^{\prime}
$$

The last equation can not be satisfies for the $L$-type and the $H$-type at the same time, which results in a contradiction. Hence, it must hold that $\bar{b}_{L} \leq \underline{b}_{H}$. Moreover, statement [ii] is obvious.

Lemma 4.3. If $h>\frac{L}{2}$, a bidder's infimum bid is zero. If $h \leq \frac{L}{2}$, the infimum bid is $h$. 
Proof. Let $b^{*} \equiv \inf \{z \mid G(z)>0\}$ denote the infimum of a bidder's bids in a symmetric equilibrium. We first show that $b^{*}$ could only be zero or $h$. Suppose the infimum bid is $b^{*} \in(0, h)$. By Lemma 4.1, take an $\epsilon>0$ such that $G\left(b^{*}+\epsilon\right)-G\left(b^{*}\right)<b^{*}$. Then, any individual bidder $i$ could profitably move density in $\left(b^{*}, b^{*}+\epsilon\right)$ arbitrarily close to zero. For $b \in\left(b^{*}, b^{*}+\epsilon\right)$, the payment would drop by $b$. The probability of winning would drop by $G(b)-G\left(b^{*}\right)<b^{*} \leq b$. Since such a profitable deviation exists, an infimum bid of $b^{*} \in(0, h)$ cannot occur in equilibrium. Thus, only zero and $h$ are possible infimum bids in equilibrium. We analyze these two possibilities one by one below.

Consider the case in which $h>L / 2$. We employ a proof by contradition to show that a bidder's infimum bid in a symmetric equilibrium is zero. If a participating bidder bids 0 , her payoff is 0 . Suppose $b^{*}=h$. Then every bidder bids $h$ with a probability of 1 , and this results into a tie. The $L$-type's expected payoff is thus

$$
\frac{1}{2}-\frac{h}{L}<0
$$

Thus, $b^{*}=h$ cannot occur in a symmetric equilibrium when $h>L / 2$. Hence, $b^{*}=0$.

Consider the case in which $h \leq L / 2$. Suppose the infimum bid is zero. Then each bidder does not bid $h$ with a probability of one. A bid near zero must be as good as a bid of $h$ for the $L$-type. If the $L$-type bids $h$, her expected payoff is strictly higher than $\frac{1}{2}-\frac{h}{L} \geq 0$. But if there is no mass at zero, then the $L$-type receives a payoff of 0 , if she bids arbitrarily close to zero. Therefore, there must have mass at zero, resulting in a contradiction to Lemma 4.1. Hence, the infimum bid in this case is $b^{*}=h$.

Lemma 4.4. Suppose $h \in\left(\frac{L}{2},(1-p)+p L\right)$. In a symmetric equilibrium, there must be mass at $h$. There exists a constant $c<h$ such that both bidders place nonzero density on almost every $b \in(0, c]$ and zero density on almost every $b \in(c, h)$.

Proof. We first show that there is mass at $h$ in a symmetric equilibrium strategy. Suppose on the contrary there is no mass at $h$. Lemmas 4.1 and 4.3 show that the infimum bid is zero and there is no mass at zero. Suppose $h \in(L / 2, L)$. If the $L$-type bids arbitrarily close to zero, her expected payoff is 0. If she bids $h$, her expected payoff is $1-\frac{h}{L}>0$. Hence, there must be mass at $h$. Suppose $h \in[L,(1-p)+p L)$. Following from Lemma 4.2, $\bar{L}=\underline{b}_{H}=p L$. If the $H$-type bids $p L$, her expected payoff is $p-p L$. If she bids $h$, her expected payoff is $1-h$, which is larger than $p-p L$. Hence, again, there must be mass at $h$.

Let the mass at $h$ be $\alpha$. There must be a $b^{*} \in(0, h)$ such that the density is zero in $\left(b^{*}, h\right)$. Because for any $b$ close enough to $h$, a bidder could profitably move it to $h$ : The payment would rise by less than a little bit but the probability of winning would rise by at least $\frac{\alpha}{2}$.

Let $c$ denote the smallest $b^{*} \in(0, h)$ such that the density in $\left(b^{*}, h\right)$ is zero. The density on almost every $b \in(0, c)$ must be positive. Suppose not. Let $t^{\prime}$ be the largest $t$ 
such that there is an interval $\left(s_{t}, t\right) \subset(0, c)$ in which the density is zero. Then, a bidder could profitably move density from $\left(t^{\prime}, t^{\prime}+\epsilon\right)$ down to $s_{t^{\prime}}$, for some $\epsilon>0$.

Proof of Proposition 2.1. In case 1, in which $h \geq(1-p)+p L$, the cap is not restrictive and the equilibrium is the same as that of Lu et al. (2018).

In case 5 , in which $h \leq L / 2$, following from Lemma 4.3 both bidders bid $h$ with a probability of one.

In the following, we consider cases $2-4$, in which $h \in(L / 2,(1-p)+p L)$. Following from Lemma 4.4, we only need to pin down the critical value of $c$.

Step 1. Suppose $c \geq p L$, we show that $\bar{b}_{L}=p L$. Suppose $\bar{b}_{L}>p L$. Following from Lemma 4.2, we must have in this case

$$
G(p L)<p
$$

However, in an equilibrium, the $L$-type's expected utility from bidding $x \leq \min \left\{\bar{b}_{L}, c\right\}$ is 0 :

$$
\begin{aligned}
E U_{L}(x)=E U_{L}(0) & \Leftrightarrow G(x)-x / L=0 \\
& \Leftrightarrow G(x)=x / L \\
& \Rightarrow G(p L)=p,
\end{aligned}
$$

resulting in a contradiction. Thus, if $c \geq p L$, then $\bar{b}_{L}=p L$.

Step 2 (Case 2). Suppose $c \in(p L,(1-p)+p L)$. We show below that in this case $h \in((1-p) / 2+p L,(1-p)+p L)$ and $c=2 h-[(1-p)+p L]$. First, following from step 1 and Lemma 4.4, we obtain that $\underline{b}_{H}\left(=\bar{b}_{L}\right)=p L$. Second, let us identify the value of $c$. The $H$-type's expected utility from bidding $x$ for all $x \in[p L, c] \cup\{h\}$ is the same as that from bidding $p L$. Thus, for all $x \in[p L, c]$,

$$
\begin{aligned}
E U_{H}(x)=E U_{H}(p L) & \Leftrightarrow G(x)-x=G(p L)-p L \\
& \Leftrightarrow G(x)=x+p(1-L),
\end{aligned}
$$

where equality 4 is obtained by substituting equation 2 to equation 3. Moreover,

$$
\begin{aligned}
E U_{H}(c)=E U_{H}(h) & \Leftrightarrow G(c)-c=\left[G(c)+\frac{1-G(c)}{2}\right]-h \\
& \Leftrightarrow c=2 h-[(1-p)+p L] .
\end{aligned}
$$

Then,

$$
\begin{aligned}
& c>p L \Leftrightarrow 2 h-[(1-p)+p L]-p L>p L \Leftrightarrow h>(1-p) / 2+p L ; \\
& c<(1-p)+p L \Leftrightarrow 2 h-[(1-p)+p L]<(1-p)+p L \Leftrightarrow h<(1-p)+p L .
\end{aligned}
$$


Hence, $c=2 h-[(1-p)+p L]$ iff $h \in((1-p) / 2+p L,(1-p)+p L)$. In this case, there is a unique symmetric equilibrium, in which the $L$-type bids according to a uniform distribution over $[0, p L]$ with a probability of 1 , whereas the $H$-type bids $h$ with a probability of $\frac{1-G(c)}{1-p}$ and bids according to a uniform distribution over $[p L, c]$ with a probability of $\frac{G(c)-G(p L)}{1-p}$.

Step 3 (Case 3). Suppose $c=p L$. We show below that $h \in[(1+p) L / 2,(1-p) / 2+p L]$. From step 1 , we know that $\bar{b}_{L}=p L$. From Lemmas 4.1 and $4.2, \underline{b}_{H}=h$ (otherwise, there would be mass at $c$ ). Moreover, the $L$-type should prefer bidding 0 to bidding $h$. Thus,

$$
\begin{aligned}
E U_{L}(h) \leq E U_{L}(0) & \Leftrightarrow G(c)+[1-G(c)] / 2-h / L \leq 0 \\
& \Leftrightarrow(1+p) / 2-h / L \leq 0 \\
& \Leftrightarrow h \geq(1-p) L / 2
\end{aligned}
$$

The $H$-type should prefer bidding $h$ to bidding $c$. Thus,

$$
\begin{aligned}
E U_{H}(c) \leq E U_{H}(h) & \Leftrightarrow G(c)-c \leq\left[G(c)+\frac{1-G(c)}{2}\right]-h \\
& \Leftrightarrow c \leq 2 h-[(1-p)+p L] \\
& \Leftrightarrow p L \leq 2 h-[(1-p)+p L] \\
& \Leftrightarrow h \leq(1-p) / 2+p L .
\end{aligned}
$$

Hence, $c=p L$ iff $h \in[(1+p) L / 2,(1-p) / 2+p L]$. In this case, there is a unique symmetric equilibrium, in which the $L$-type bids according to a uniform distribution over $[0, p L]$ with a probability of 1 , whereas the $H$-type bids $h$ with a probability of 1 .

Step 4 (Case 4). Suppose $c \in(0, p L)$. We show below that $h \in(L / 2,(1+p) L / 2)$. First, let us show that $\underline{b}_{H}\left(=\bar{b}_{L}\right)=h$. Suppose $\underline{b}_{H}<h$, then $\bar{b}_{L}=\underline{b}_{H}<c<p L$, and a contradiction can be shown below.

$$
\begin{aligned}
0 & =E U_{H}(c)-E U_{H}(h) \\
& =[G(c)-c]-\left[\frac{1+G(c)}{2}-h\right] \\
& =h-\left[\frac{1-G(c)}{2}+c\right] \\
& >h-[(1-p) / 2+p L] ; \\
0 & =E U_{H}\left(\bar{b}_{L}\right)-E U_{H}(h) \\
& =\left[G\left(\bar{b}_{L}\right)-\bar{b}_{L}\right]-\left[\frac{1+G(c)}{2}-h\right] \\
& <(p-p L)-[(1+p) / 2-h]
\end{aligned}
$$




$$
=h-[(1-p) / 2+p L] .
$$

Hence, there must be $\underline{b}_{H}=h$.

Second, suppose $\bar{b}_{L}<h$. Following from the first part, then $\bar{b}_{L}=c<h=\underline{b}_{H}$, and a contradiction can be shown below. For all $x \in[0, c]$,

$$
\begin{aligned}
E U_{L}(x)=E U_{L}(0) & \Rightarrow E U_{L}(c)=E U_{L}(0) \\
& \Leftrightarrow G(c)-c / L=0 \\
& \Rightarrow p L-c=0
\end{aligned}
$$

which contradicts to $c<p L$. Hence, there must be $\bar{b}_{L}=h$. Moreover, in this case, the $L$-type's expected utility from bidding $x$ for all $x \in[0, c] \cup\{h\}$ is 0 . Thus,

$$
\begin{aligned}
E U_{L}(x)=E U_{L}(0) & \Leftrightarrow G(x)-x / L=0 \\
& \Leftrightarrow G(x)=x / L \\
& \Rightarrow G(c)=c / L . \\
E U_{L}(0)=E U_{L}(h) & \Leftrightarrow 0=\left[G(c)+\frac{1-G(c)}{2}\right]-h / L \\
& \Leftrightarrow 0=\frac{1+G(c)}{2}-h / L \\
& \Leftrightarrow c=2 h-L,
\end{aligned}
$$

where equation 7 is obtained by substituting equation 5 to equation 6 . Then,

$$
\begin{aligned}
& c<p L \Leftrightarrow(2 h-L)<p L \Leftrightarrow h<(1+p) L / 2 ; \\
& c>0 \Leftrightarrow(2 h-L)>0 \Leftrightarrow h<L / 2 .
\end{aligned}
$$

Hence, $c=2 h-L$ iff $h \in(L / 2,(1+p) L / 2)$. In this case, there is a unique symmetric equilibrium, in which the $L$-type bids $h$ with a probability of $1-\frac{G(c)}{p}$ and bids according to a uniform distribution over $[0, p L]$ with a probability of $\frac{G(c)}{p}$, whereas the $H$-type bids $h$ with a probability of 1 . 


\section{Appendix B: Revenue comparison between $C C C$ and $D D D$}

For convenience, let us denote the expected revenue in each region of the bid cap under policy $C C C$ by

$$
E R^{C C C}(h)= \begin{cases}R_{1}^{C} & \text { if } h \leq L / 2, \\ R_{2}^{C} & \text { if } h \in(L / 2,(1+p) L / 2), \\ R_{3}^{C} & \text { if } h \in[(1+p) L / 2,(1-p) / 2+p L), \\ R_{4}^{C} & \text { if } h \geq(1-p) / 2+p L\end{cases}
$$

and that in each region of the bid cap under policy $D D D$ by

$$
E R^{D D D}(h)= \begin{cases}R_{1}^{D} & \text { if } h \leq L / 2, \\ R_{2}^{D} & \text { if } h \in(L / 2,1 / 2) \\ R_{3}^{D} & \text { if } h \geq 1 / 2 .\end{cases}
$$

\section{Lemma 4.5.}

$i$ Suppose $h>\frac{L}{2} . R_{2}^{D}(h)<(=) R_{2}^{C}(h)$ iff $p>(=) \frac{h-\frac{L}{2}}{h-\frac{L}{2} \cdot L}$.

ii $R_{2}^{D}(h)<(=) R_{3}^{C}(h)$ iff $h>(=) \frac{L}{2}(1+L)$.

iii $R_{3}^{D}(h)<(=) R_{2}^{C}(h)$ iff $p>(=) \frac{1}{1+L}$.

iv $R_{3}^{D}(h)<(=) R_{3}^{C}(h)$ iff $h>(=) \frac{L}{2}\left(1+\frac{1}{1+L}\right)-\frac{1+L}{2}\left(\frac{1}{1+L}-p\right)\left(L-\frac{1}{1+L}\right)$

$v E R^{D}(h)<R_{4}^{C}(h)$ for all $h \geq \frac{L}{2}$.

Proof. Immediate from corollaries 2.1 and 2.2.

Lemma 4.6. Corollary 2.1 can be rewritten as follows. Suppose $L>\frac{1}{2}$.

1. If $h \geq L$,

2. If $L>h \geq \frac{1}{2}$,

3. If $\frac{1}{2}>h>\frac{L}{2}$

$$
E R^{C C C}(h)=R_{4}^{C}(h) .
$$$$
E R^{C C C}(h)= \begin{cases}R_{4}^{C}(h) & \text { if } p \leq \frac{h-1 / 2}{L-1 / 2} \\ R_{3}^{C}(h) & \text { if } p \in\left(\frac{h-1 / 2}{L-1 / 2}, \frac{h-L / 2}{L / 2}\right] \\ R_{2}^{C}(h) & \text { if } p>\frac{h-L / 2}{L / 2} .\end{cases}
$$$$
E R^{C C C}(h)= \begin{cases}R_{3}^{C}(h) & \text { if } p \leq \frac{h-L / 2}{L / 2} \\ R_{2}^{C}(h) & \text { if } p>\frac{h-L / 2}{L / 2}\end{cases}
$$

Suppose $\frac{1}{2} \geq L$.
1. If $h \geq \frac{1}{2}$,
$E R^{C C C}(h)=R_{4}^{C}(h)$. 

2. If $\frac{1}{2}>h \geq L$,
$E R^{C C C}(h)= \begin{cases}R_{4}^{C}(h) & \text { if } p \leq \frac{h-1 / 2}{L-1 / 2} \\ R_{3}^{C}(h) & \text { if } p>\frac{h-1 / 2}{L-1 / 2} .\end{cases}$
3. If $L>h>\frac{L}{2}$,
$E R^{C C C}(h)= \begin{cases}R_{3}^{C}(h) & \text { if } p \leq \frac{h-L / 2}{L / 2} \\ R_{2}^{C}(h) & \text { if } p>\frac{h-L / 2}{L / 2} .\end{cases}$

Proof of Theorem 2.1.

Step 1. First, note that $\frac{1}{1+L} \geq L \Leftrightarrow \frac{1}{2}-\frac{L}{2}(1+L)$. Let us make three scenario distinctions:

Scenario $1\left[L<\frac{1}{2}\right.$ (which implied $\left.\left.\frac{1}{1+L} \geq L\right)\right]: \frac{1}{2} \geq L>\frac{L}{2}(1+L)>\frac{L}{2}$.

Scenario $2\left[L>\frac{1}{2}\right.$ and $\left.\frac{1}{1+L} \geq L\right]: L>\frac{1}{2} \geq \frac{L}{2}(1+L)>\frac{L}{2}$.

Scenario $3\left[\frac{1}{1+L} \leq L\right.$ (which implies $\left.\left.L>\frac{1}{2}\right)\right]: L>\frac{L}{2}\left(1+\frac{1}{1+L}\right)>\frac{1}{2}>\frac{L}{2}$.

Second, let us divide the range of $h$ into five regions for each scenario below.

Table 1: Division of the range of the bid cap in each case

\begin{tabular}{|c|c|c|c|c|c|}
\hline & Region 0 & Region 1 & Region 2 & Region 3 & Region 4 \\
\hline Scenario 1 & {$[0, L / 2]$} & $\left(L / 2, \frac{L}{2}(1+L)\right]$ & $\left(\frac{L}{2}(1+L), L\right]$ & $(L, 1 / 2]$ & $(1 / 2,+\infty)$ \\
\hline Scenario 2 & {$[0, L / 2]$} & $\left(L / 2, \frac{L}{2}(1+L)\right]$ & $\left(\frac{L}{2}(1+L), 1 / 2\right]$ & $(1 / 2, L]$ & $(L,+\infty)$ \\
\hline Scenario 3 & {$[0, L / 2]$} & $(L / 2,1 / 2]$ & $\left(1 / 2, \frac{L}{2}\left(1+\frac{1}{1+L}\right)\right]$ & $\left(\frac{L}{2}\left(1+\frac{1}{1+L}\right), L\right]$ & $(L,+\infty)$ \\
\hline
\end{tabular}

To prove the main result, we will apply lemmas 4.5 and 4.6 to all these 15 cases.

Step 2. We analyze the cases in which $h \geq \frac{L}{2}(1+K)$ and show that in these cases $E R^{D D D}(h)<E R^{C C C}(h)$. First, in region 4 of scenarios 1, 2, and 3, $E R^{C C C}(h)=$ $R_{4}^{C}(h)>E R^{D D D}(h)$. Second, in region 3 of scenarios 2 and 3 ,

$$
E R^{D D D}(h)=R_{3}^{D}(h)<E R^{C C C}(h)= \begin{cases}R_{4}^{C}(h) & \text { if } p \leq \frac{h-1 / 2}{L-1 / 2} \\ R_{3}^{C}(h) & \text { if } p \in\left(\frac{h-1 / 2}{L-1 / 2}, \frac{h-L / 2}{L / 2}\right] \\ R_{2}^{C}(h) & \text { if } p>\frac{h-L / 2}{L / 2}\left(>L \geq \frac{1}{1+L}\right) .\end{cases}
$$

Third, in region 2 of scenarios 1 and 2 ,

$$
E R^{D D D}(h)=R_{2}^{D}(h)<E R^{C C C}(h)= \begin{cases}R_{3}^{C}(h) & \text { if } p \leq \frac{h-L / 2}{L / 2} \\ R_{2}^{C}(h) & \text { if } p>\frac{h-L / 2}{L / 2}\end{cases}
$$

Last, in region 3 of scenario 1 ,

$$
E R^{D D D}(h)=R_{2}^{D}(h)<E R^{C C C}(h)= \begin{cases}R_{4}^{C}(h) & \text { if } p \leq \frac{h-1 / 2}{L-1 / 2} \\ R_{3}^{C}(h) & \text { if } p>\frac{h-1 / 2}{L-1 / 2}\end{cases}
$$

Step 3. We analyze the cases in which $h \in\left(\frac{L}{2}, \frac{L}{2}(1+K)\right]$ and derive the necessary and sufficient conditions for $E R^{D D D}(h)>E R^{C C C}(h)$. [i]. In region 1 of all scenarios 1 , 
2 , and 3 ,

$$
E R^{D D D}(h)=R_{2}^{D}(h) \begin{cases}>E R^{C C C}(h)=R_{3}^{C}(h) & \text { if } p \leq \frac{h-L / 2}{L / 2} \\ >E R^{C C C}(h)=R_{2}^{C}(h) & \text { if } p \in\left(\frac{h-L / 2}{L / 2}, \frac{h-L / 2}{h-L / 2 \cdot L}\right) \\ =E R^{C C C}(h)=R_{2}^{C}(h) & \text { if } p=\frac{h-L / 2}{h-L / 2 \cdot L} \\ <E R^{C C C}(h)=R_{2}^{C}(h) & \text { if } p>\frac{h-L / 2}{h-L / 2 \cdot L} .\end{cases}
$$

$E R^{D D D}(h)>E R^{C C C}(h)$ iff $p<\frac{h-L / 2}{h-L / 2 \cdot L}\left(\right.$ or $\left.\left(\frac{L}{2}<\right) \frac{L}{2} \cdot \frac{1-p L}{1-p}<h\right)$. Region 1 of scenarios 1 and 2 also requires $\frac{L}{2}<h \leq \frac{L}{2}(1+L)$. Note that $\left(\frac{L}{2} \cdot \frac{1-p L}{1-p}, \frac{L}{2}(1+L)\right]$ is non-empty iff $p<L$. Also note that $\frac{h-L / 2}{h-L / 2 \cdot L}=L$ iff $h=\frac{L}{2}(1+L)$ Region 1 of scenario 3 requires $\frac{L}{2}<h \leq \frac{1}{2}$. Note that $\left(\frac{L}{2} \cdot \frac{1-p L}{1-p}, \frac{1}{2}\right]$ is non-empty iff $p<\frac{1}{1+L}$. Hence we can rewrite the above result as follows:

1. Suppose $K=L$. $E R^{D D D}(h)>E R^{C C C}(h)$ iff $p<K$ and $\frac{L}{2} \cdot \frac{1-p L}{1-p}<h<\frac{L}{2}(1+L)$.

2. Suppose $K=\frac{1}{1+L}$ and $h \in\left(\frac{L}{2}, \frac{1}{2}\right] . E R^{D D D}(h)>E R^{C C C}(h)$ iff $p<K$ and $\frac{L}{2} \cdot \frac{1-p L}{1-p}<$ $h \leq \frac{1}{2}$.

[ii]. In region 2 of scenario $3,^{15}$

$$
E R^{D D D}(h)=R_{3}^{D}(h) \begin{cases}<E R^{C}(h)=R_{4}^{C}(h) & \text { if } p \leq \frac{h-1 / 2}{L-1 / 2} \\ <E R^{C}(h)=R_{3}^{C}(h) & \text { if } p \in\left(\frac{h-1 / 2}{L-1 / 2}, \frac{h-1 / 2}{\frac{L}{2}(1+L)-1 / 2}\right) \\ =E R^{C}(h)=R_{3}^{C}(h) & \text { if } p=\frac{h-1 / 2}{\frac{L}{2}(1+L)-1 / 2} \\ >E R^{C}(h)=R_{3}^{C}(h) & \text { if } p \in\left(\frac{h-1 / 2}{\frac{L}{2}(1+L)-1 / 2}, \frac{h-L / 2}{L / 2}\right] \\ >E R^{C}(h)=R_{2}^{C}(h) & \text { if } p \in\left(\frac{h-L / 2}{L / 2}, \frac{1}{1+L}\right) \\ =E R^{C}(h)=R_{2}^{C}(h) & \text { if } p=\frac{1}{1+L} \\ <E R^{C}(h)=R_{2}^{C}(h) & \text { if } p>\frac{1}{1+L} .\end{cases}
$$

In this case $L \geq \frac{1}{1+L}$ and $1 / 2<h \leq \frac{L}{2}\left(1+\frac{1}{1+L}\right)$, and $E R^{D D D}(h)>E R^{C C C}(h)$ iff $\frac{h-1 / 2}{\frac{L}{2}(1+L)-1 / 2}<p<\frac{1}{1+L}$. This can be rewritten as follows: Suppose $K=\frac{1}{1+L}$ and $h>\frac{1}{2}$. $E R^{D D D}(h)>E R^{C C C}(h)$ iff $p<K$ and $\frac{1}{2}<h<\frac{L}{2}(1+K)-\frac{1+L}{2}(K-p)(L-K)$.

[i] and [ii] together yield the desired result.

\section{Appendix C: Symmetric equilibrium under policy $D C C$ when $C$ occurs}

With a little abuse of notation, let $F_{L}(\cdot)$ and $F_{H}(\cdot)$ be the $L$-type's and the $H$-types's distribution of bids, respectively in a symmetric equilibrium. Let $\underline{b}_{L} \equiv \inf \left\{z \mid F_{L}(z)>\right.$

\footnotetext{
${ }^{15}$ Note that given that $h \geq 1 / 2$ and $L \geq 1 / 2, h \leq \frac{L}{2}\left(1+\frac{1}{1+L}\right) \Leftrightarrow \frac{h-L / 2}{L / 2} \leq \frac{1}{1+L} \Leftrightarrow \frac{h-1 / 2}{(1+L) L / 2-1 / 2} \leq$ $\frac{h-L / 2}{L / 2} \Leftrightarrow \frac{h-1 / 2}{L-1 / 2} \leq \frac{h-1 / 2}{(1+L) L / 2-1 / 2}$.
} 
$0\}$ and $\bar{b}_{L} \equiv \inf \left\{z \mid F_{L}(z)=1\right\}$ denote the $L$-type's infimum bid and superimum bid, respectively. Let $\underline{b}_{H} \equiv \inf \left\{z \mid F_{H}(z)>0\right\}$ and $\bar{b}_{H} \equiv \inf \left\{z \mid F_{H}(z)=1\right\}$ denote the $H$-type's infimum bid and superimum bid, respectively.

Lemma 4.7. $\underline{b}_{L} \leq \underline{b}_{H} \leq \bar{b}_{H} \leq \bar{b}_{L}$.

Proof. Suppose $\underline{b}_{L}>\underline{b}_{H}$. Then, considering that the $H$-type knows that her opponent is exactly the $L$-type, the $H$-type could profitably move density from $\left[\underline{b}_{H}, \underline{b}_{H}+\epsilon\right)$ up to slightly below $\underline{b}_{L}$, for some $\epsilon>0$.

Suppose $\bar{b}_{L}<\bar{b}_{H}$. Then, considering that the $H$-type knows that her opponent is exactly the $L$-type, the $H$-type could profitably move density from $\left[\bar{b}_{H}-\epsilon, \bar{b}_{H}\right]$ down to slightly above $\bar{b}_{L}$, for some $\epsilon>0$.

Lemma 4.8. No bidder has mass at any bid $b \in[0, h)$.

Proof. This is similar to Lemma 4.1.

Lemma 4.9. If $h>\frac{L}{2}$, then $\underline{b}_{L}=0$. If $h \leq \frac{L}{2}$, both types bid $h$ with a probability of 1 .

Proof. This is similar to Lemma 4.3.

Lemma 4.10. Suppose $h \in(L / 2, L)$. There exists a constant $c<h$ such that: the $L$-type has a mass of $\alpha_{L} \in(0,1)$ at $h$, places nonzero density on almost every $b \in[0, c]$, and places zero density on almost every $b \in(c, h)$; the $H$-type has a mass of $\alpha_{H} \in(0,1]$ at $h$. If $\alpha_{H}<1$, there is an $\underline{x}<c$ such that the $H$-type places nonzero density on almost every $b \in[\underline{x}, c]$ and places zero density on almost every $b \in(c, h)$.

Proof. We need to show $\alpha_{L} \in(0,1)$ and $\alpha_{H} \in(0,1]$.

Step 1. Suppose either $\alpha_{L}>0$ or $\alpha_{H}>0$. There must be a $b^{*} \in(0, h)$ such that the density of $F_{L}$ is almost sure zero in $\left(b^{*}, h\right)$. Because for any $b$ close enough to $h$, the $L$-type could profitably move it to $h$ : Her payment would rise by less than a little bit but her probability of winning would rise by at least $\frac{p \alpha_{L}+(1-p) \alpha_{H}}{2}$.

Let $c$ denote the smallest $b^{*} \in(0, h)$ such that the density of $F_{L}$ in $\left(b^{*}, h\right)$ is almost sure zero. The density on almost every $b \in(0, c]$ must be positive. Suppose not. Let $t^{\prime}$ be the largest $t$ such that there is an interval $\left(s_{t}, t\right) \subset(0, c]$ in which the density is zero. First, the $H$-type would also place zero density on almost every point in $\left(s_{t}, t\right)$, because the $H$-type knows that her opponent is the $L$-type and can profitably move density from $\left(s_{t}, t\right)$ to $s_{t}$. Next, the $L$-type could then profitably move density from $(t, t+\epsilon)$ down to $s_{t}$, for some $\epsilon>0$.

The $H$-type also place zero density on almost every $b \in(c, h)$, again, because the $H$-type knows that her opponent is the $L$-type.

Step 2. We will show that $\alpha_{H}, \alpha_{L}>0$, by ruling out the other three cases. 
First, it is not possible that $\alpha_{H}>0=\alpha_{L}$, becasue the $H$-type knows that her opponent is the $L$-type and can profitably move weight slightly from $h$ down to slightly below $h$.

Second, suppose $\alpha_{H}=\alpha_{L}=0$. Lemmas 4.8 and 4.9 show that the $L$-type's infimum bid is zero and there is no mass at zero. Suppose $h \in(L / 2, L)$. If the $L$-type bids arbitrarily close to zero, her expected payoff is approximately 0 . If she bids $h$, her expected payoff is $1-\frac{h}{L}>0$. Hence, the $L$-type must have mass at $h$, resulting in a contradiction.

Third, suppose $\alpha_{H}=0<\alpha_{L}$. Then, $\underline{b}_{H}<\bar{b}_{H} \leq c$. The $L$-type is indifferent between bidding $\underline{b}_{H}$ and $h$ :

$E U_{L}\left(\underline{b}_{H}\right)=E U_{L}(h) \Leftrightarrow p F_{L}\left(\underline{b}_{H}\right)-\underline{b}_{H} / L=p F_{L}(c)+(1-p) F_{H}(c)+\left[1-F_{L}(c)\right] / 2-h / L$.

However, the $H$-type strictly prefers bidding $h$ to bidding $\bar{b}_{H}$ :

$$
E U_{H}\left(\underline{b}_{H}\right)=F_{L}\left(\underline{b}_{H}\right)-\underline{b}_{H}<F_{L}(c)+\left[1-F_{L}(c)\right] / 2-h=E U_{H}(h)
$$

Thus, the $H$-type could then profitably move density from $\left[\underline{b}_{H}, \underline{b}_{H}+\epsilon\right)$, for some $\epsilon>0$, up to $h$, resulting in a contradiction.

Step 3. Suppose $\alpha_{H}<1$. We will show that the $H$-type places positive density on almost every $b \in\left[\underline{b}_{H}, c\right]$ (and zero density on almost every $b \in\left[0, \underline{b}_{H}\right)$ ). Suppose not. Let $t^{\prime}$ be the lowest $t$ such that there is an interval $\left(t, s_{t}\right) \subset\left[\underline{b}_{H}, c\right)$ in which the density is almost sure zero. The $L$-type is indifferent between bidding $t$ and $s_{t}$ :

$$
E U_{L}(t)=E U_{L}\left(s_{t}\right) \Leftrightarrow p F_{L}(t)+(1-p) F_{H}(t)-t / L=p F_{L}\left(s_{t}\right)+(1-p) F_{H}(t)-s_{t} / L
$$

However, the $H$-type strictly prefers bidding $s_{t}$ to bidding $t$ :

$$
E U_{H}(t)=F_{L}(t)-t<F_{L}\left(s_{t}\right)-s_{t}=E U_{H}\left(s_{t}\right) .
$$

The $H$-type could then profitably move density from $\left[\underline{b}_{H}, t\right]$ up to $s_{t}$.

Proof of Proposition 3.1. In case 1 , in which $h \geq L$, the cap is not restrictive and the equilibrium is the same as that of $\mathrm{Lu}$ et al. (2018).

In case 4 , in which $h \leq L / 2$, following from Lemma 4.3 both bidders bid $h$ with a probability of one.

In the following, we consider cases 2 and 3 , in which $h \in(L / 2, L)$. In case 2 the $H$-type bids $h$ with a probability less than 1 , i.e., $\alpha_{H}<1$, and in case 3 in the $H$-type bids $h$ with a probability of 1 , i.e., $\alpha_{H}=1$.

For case 2, following from Lemma 4.10, we only need to solve a linear equation system and determine the values of $\underline{x}, c, F_{L}(\underline{x}), F_{L}(c)$, and $F_{H}(c)$. The expected utilities of the 
$L$-type and the $H$-type are given, respectively, below:

$$
\begin{aligned}
0=E U_{L}(x) & = \begin{cases}p F_{L}(x)-\frac{x}{L} & \text { for } x \in[0, \underline{x}] \\
p F_{L}(x)+(1-p) F_{H}(x)-\frac{x}{L} & \text { for } x \in(\underline{x}, c] \\
p F_{L}(c)+(1-p) F_{H}(c)+\frac{p\left[1-F_{L}(c)\right]+(1-p)\left[1-F_{H}(c)\right]}{2}-\frac{h}{L} & \text { for } x=h ;\end{cases} \\
E U_{H}(x) & = \begin{cases}F_{L}(x)-x & \text { for } x \in(\underline{x}, c] \\
F_{L}(c)+\frac{p\left[1-F_{L}(c)\right]+(1-p)\left[1-F_{H}(c)\right]}{2}-\frac{h}{L} & \text { for } x=h .\end{cases}
\end{aligned}
$$

We can then write out the following five equalities with five unknowns:

$$
\begin{gathered}
p F_{L}(\underline{x})-\frac{\underline{x}}{L}=0 ; \\
p F_{L}(c)+(1-p) F_{H}(c)-\frac{c}{L}=0 ; \\
p F_{L}(c)+(1-p) F_{H}(c)+\frac{p\left[1-F_{L}(c)\right]+(1-p)\left[1-F_{H}(c)\right]}{2}-\frac{h}{L}=0 ; \\
F_{L}(\underline{x})-\underline{x}=F_{L}(c)-c=F_{L}(c)+\frac{1-F_{L}(c)}{2}-h .
\end{gathered}
$$

Solving the above five equations with five unknowns yields

$$
\begin{aligned}
\underline{x} & =\frac{p-p L}{1-p L} \cdot L ; \\
c & =2 h-L ; \\
F_{L}(\underline{x}) & =\frac{1-L}{1-p L} ; \\
F_{L}(c) & =1-2(L-h) ; \\
F_{H}(c) & =\frac{2 h(H-p L)-2(1-L) L p}{(1-p) L} .
\end{aligned}
$$

Moreover, $\alpha_{H}<1$ iff $F_{H}(c)>0$. Hence, $h>\frac{L}{2}\left(\frac{p(1-L)}{1-p L}+1\right)$. In this case, there is a unique symmetric equilibrium, in which the $L$-type bids $h$ with a probability of $1-F_{L}(c)$, bids according to a uniform distribution over $[0, \underline{x}]$ with a probability of $F_{L}(\underline{x})$, and bids according to a uniform distribution over $[\underline{x}, c]$ with a probability of $F_{L}(c)-F_{L}(\underline{x})$, whereas the $H$-type bids $h$ with a probability of $1-F_{H}(c)$ and bids according to a uniform distribution of $[\underline{x}, c]$ with a probability of $F_{H}(c)$.

For case 3, following from Lemma 4.10, we only need to solve a linear equation system and determine the values of $c, F_{L}(\underline{x})$, and $F_{L}(c)$. The expected utility of the $L$-type is given below:

$$
0=E U_{L}(x)= \begin{cases}p F_{L}(x)-\frac{x}{L} & \text { for } x \in[0, c] \\ p F_{L}(c)+(1-p) F_{H}(c)+\frac{p\left[1-F_{L}(c)\right]+(1-p)\left[1-F_{H}(c)\right]}{2}-\frac{h}{L} & \text { for } x=h .\end{cases}
$$


We can then write out the following two equalities:

$$
\begin{aligned}
p F_{L}(c)-\frac{c}{L} & =0 \\
p F_{L}(c)+\frac{p\left[1-F_{L}(c)\right]+(1-p)}{2}-\frac{h}{L} & =0 .
\end{aligned}
$$

Solving the above two equations with two unknowns yields

$$
c=2 h-L \text { and } F_{L}(c)=\frac{2(h-L / 2)}{p L} .
$$

In addition, we can get $F_{L}(x)=x / L$ for $x \in[0, c]$, and thus, if the $H$-type bids $x \in[0, c]$, her expected utility is $E U_{H}(x)=F_{L}(x)-x=x / L-x$, which is increasing in $x$. Hence, $\alpha_{H}=1$ iff

$$
\begin{aligned}
E U_{H}(h) \geq E U_{H}(c) & \Leftrightarrow F_{L}(c)+\left[1-F_{L}(c)\right] / 2-h \geq F_{L}(c)-c \\
& \Leftrightarrow \frac{1-\frac{2(h-L / 2)}{p L}}{2}-h \geq-(2 h-L) \\
& \Leftrightarrow h \leq \frac{L}{2}\left(\frac{p(1-L)}{1-p L}+1\right) .
\end{aligned}
$$

In this case, there is a unique symmetric equilibrium, in which the $L$-type bids $h$ with a probability of $1-F_{L}(c)$ and bids according to a uniform distribution over $[0, c]$ with a probability of $F_{L}(c)$, whereas the $H$-type bids $h$ with a probability of 1 .

\section{Appendix D: Symmetric equilibrium under policy $C C D$ when $C$ occurs}

Again, with a little abuse of notation, let $F_{L}(\cdot)$ and $F_{H}(\cdot)$ be the $L$-type's and the $H$ types's distribution of bids, respectively in a symmetric equilibrium. Let $\underline{b}_{L} \equiv \inf \left\{z \mid F_{L}(z)>\right.$ $0\}$ and $\bar{b}_{L} \equiv \inf \left\{z \mid F_{L}(z)=1\right\}$ denote the $L$-type's infimum bid and superimum bid, respectively. Let $\underline{b}_{H} \equiv \inf \left\{z \mid F_{H}(z)>0\right\}$ and $\bar{b}_{H} \equiv \inf \left\{z \mid F_{H}(z)=1\right\}$ denote the $H$-type's infimum bid and superimum bid, respectively.

Lemma 4.11. In a (symmetric) equilibrium bidding strategy, there is no mass point at any bid $b \in(0, h)$ and the $H$-type has no mass at 0 .

Proof. The first half of the claim is similar to Lemma 4.1. For the second half, suppose $F_{H}(0)>0$, then the $L$-type has no mass at 0 , because the $L$-type knows that her opponent is exactly the $H$-type and prefers slightly bidding above 0 to bidding 0 . Then the $H$-type could profitably move weight from 0 to slightly above 0 .

Lemma 4.12. If $h \leq \frac{L}{2}$, both types bid $h$ with a probability of 1 .

Proof. This is similar to Lemma 4.3 . 


\section{Lemma 4.13.}

i. Consider the case in which $L \leq 1-p$.

(a) Suppose $h \in((1-p) / 2,1-p)$. There exists a constant $c<h$ such that the $H$-type has a mass of $\alpha_{H} \in(0,1)$ at $h$, places nonzero density on almost every $b \in[0, c]$, and places zero density on almost every $b \in(c, h)$. The L-type bids 0 with a probability of 1 .

(b) Suppose $h \in(L / 2,(1-p) / 2]$. The $H$-type bids $h$ with a probability of 1 . The L-type bids 0 with a probability of 1 .

ii. Consider the case in which $L>1-p$. Suppose $h \in(L / 2, L)$. There exists a constant $c<h$ such that the $H$-type has a mass of $\alpha_{H} \in(0,1)$ at $h$, places nonzero density on almost every $b \in[0, c]$, and places zero density on almost every $b \in(c, h)$. The $L$-type has a mass $\alpha_{L} \in(0,1)$ at $h$ and a mass $F_{L}(0) \in(0,1)$ at 0 , places nonzero density on almost every $b \in(0, c]$, and places zero density on almost every $b \in(c, h)$.

Proof. We need to show $\alpha_{L} \in(0,1)$ and $\alpha_{H} \in(0,1]$.

Step 1. Suppose either $\alpha_{L}>0$ or $\alpha_{H}>0$. There must be a $b^{*} \in[0, h)$ such that the density of $F_{H}$ on almost every $b \in\left(b^{*}, h\right)$ is zero. Because for any $b$ close enough to $h$, the $H$-type could profitably move the density around $b$ up to $h$ : Her payment would rise by less than a little bit but her probability of winning would rise by at least $\frac{p \alpha_{L}+(1-p) \alpha_{H}}{2}$.

Step 2. Let $c$ denote the smallest $b^{*} \in[0, h)$ such that the density of $F_{H}$ on almost every $b \in\left(b^{*}, h\right)$ is zero. If $c>0$, the density on almost every $b \in[0, c)$ must be positive. Suppose not. Let $t^{\prime}$ be the largest $t$ such that there is an interval $\left(s_{t}, t\right) \subset(0, c)$ in which the density is almost sure zero. First, the $L$-type bidder would also place zero density at almost every point in $\left(s_{t^{\prime}}, t^{\prime}\right)$, because the $L$-type knows that her opponent is the $H$ type and can profitably move density from $\left(s_{t^{\prime}}, t^{\prime}\right)$ to $s_{t^{\prime}}$. Next, the $H$-type could then profitably move density from $\left(t^{\prime}, t^{\prime}+\epsilon\right)$ down to $s_{t^{\prime}}$, for some $\epsilon>0$.

The $L$-type also places zero density on almost every $b \in(c, h)$, again, because the $L$-type knows that her opponent is the $H$-type.

Step 3. Suppose $\alpha_{H}=1$, we show that $F_{L}(0)=1$. In this case, $c=0$. We only need to show that the $L$-type has no incentive to bid $h$, which is true because her expected payoff is negative if she bids $h$ :

$$
E U_{L}(h)=1 / 2-\frac{h}{L}<0 .
$$

Hence, in this case the $L$-type bids 0 with a probability of 1 .

Step 4. We show that $F_{L}(0)>0$. Step 3 has shown for the case in which $\alpha_{H}=1$. We show that for the case in which $\alpha_{H}<1$ below. Suppose $F_{L}(0)=0$. Then, from step 
2, the infimum bid of the $H$-type is $\underline{b}_{H}=0$, and we have $c>0$. Thus, the $H$-type's expected payoff is 0 , the expected payoff from bidding 0 .

$$
\begin{aligned}
E U_{H}(c)=0 & \Rightarrow(1-p) F_{H}(c)+p F_{L}(c)=c \\
E U_{H}(h)=0 & \Rightarrow(1-p) F_{H}(c)+p F_{L}(c)+\frac{(1-p)\left[1-F_{H}(c)\right]+p\left[1-F_{L}(c)\right]}{2}-h=0 \\
& \Rightarrow \frac{(1-p)\left[1+F_{H}(c)\right]+p\left[1+F_{L}(c)\right]}{2}-h=0 \\
& \Rightarrow \frac{1+c}{2}-h=0 \\
& \Rightarrow c=2 h-1 .
\end{aligned}
$$

$c>0$ implies $h>1 / 2$. The expected utility of the $L$-type is then:

$$
E U_{L}(h)=\frac{1-F_{H}(c)}{2}=\frac{2-p-2 h}{2(1-p)}-h=\frac{(2-p)(1-2 h)}{2(1-p)}<0 .
$$

Thus, the $L$-type strictly prefers bidding 0 and should have a mass at 0 , resulting in a contradiction.

Step 5. Given that $\alpha_{H} \in(0,1)$, we calculate the bidding strategy of the $H$-type below. The $H$-type's expected utility is $p F_{L}(0)$ : for all $x \in(0, c] \cup\{h\}$,

$$
\begin{aligned}
E U_{H}(x)=p F_{L}(0) & \Rightarrow p F_{L}(x)+(1-p) F_{H}(x)-x=p F_{L}(0) \\
& \Rightarrow F_{H}(x)=\frac{x}{1-p}-\frac{p\left[F_{L}(x)-F_{L}(0)\right]}{1-p} ; \\
E U_{H}(h)=p F_{L}(0) & \Rightarrow p F_{L}(c)+(1-p) F_{H}(c)+\frac{p\left[1-F_{L}(c)\right]+(1-p)\left[1-F_{H}(c)\right]}{2}-h=p F_{L}(0) \\
& \Rightarrow \frac{1+F_{H}(c)}{2}=\frac{h}{1-p}-\frac{p\left[1+F_{L}(c)-2 F_{L}(0)\right]}{2(1-p)}
\end{aligned}
$$

Step 6. Consider case $i$ in which $L \leq 1-p$. We show that: (1) the $L$-type bids 0 with a probability of 1 and (2) the $H$-type bids $h$ with a probability of 1 iff $h \in(L / 2,(1-p) / 2]$.

Suppose $h \in(L / 2,1-p)$. First, it is not possible that $\alpha_{L}>0=\alpha_{H}$, becasue the $L$-type knows that her opponent is the $H$-type and can profitably move weight down slightly from $h$.

Second, it is not possible that $\alpha_{L}=\alpha_{H}=0$. Suppose $\alpha_{L}=\alpha_{H}=0$. The expected payoff of the $H$-type is $1-h$ if she bids $h$, whereas her expected payoff is at most $p F_{L}\left(\underline{b}_{H}\right)-\underline{b}_{H}<p$ if she bids $\underline{b}_{H}$. Since $1-h>p$, the $H$-type can profitably move weight from $\left[\underline{b}_{H}, \underline{b}_{H}+\epsilon\right]$ up to $h$, for some $\epsilon>0$. Hence, we must have $\alpha_{H}>0$, resulting in a contradiction.

Third, we show that the $L$-type bids 0 with a probability of 1 . As shown in step 3, this is true if $\alpha_{H}=1$. We only need to consider the case in which $\alpha_{H} \in(0,1)$. In this 
case, $\underline{b}_{H}=0$ and $c>0$. The $L$-type has no incentive to bid $h$ with a positive probability, because doing so leads to a negative payoff:

$$
\begin{aligned}
E U_{L}(h) & =F_{H}(c)+\frac{1-F_{H}(c)}{2}-\frac{h}{L} \\
& =h\left(\frac{1}{1-p}-\frac{1}{L}\right)-\frac{p\left[1+F_{L}(c)-2 F_{L}(0)\right]}{2(1-p)} \\
& <0 .
\end{aligned}
$$

The $L$-type also has no incentive to place positive density on a non-null set in $(0, c]$, because for any $x \in(0, c]$ such that $F_{L}(x)>F_{L}(0)$,

$$
\begin{aligned}
E U_{L}(x) & =F_{H}(x)-\frac{x}{L} \\
& =x\left(\frac{1}{1-p}-\frac{1}{L}\right)-\frac{p\left[F_{L}(x)-F_{L}(0)\right]}{1-p} \\
& <0 .
\end{aligned}
$$

Hence, in this case the $L$-type bids 0 with a probability of 1 as well.

Fourth, $\alpha_{H}<1$ iff $c>0$ (or equivalently $F_{H}(c)>0$ ). Since $F_{L}(0)=1$, following from equation 9:

$$
\frac{1}{2}<\frac{h}{1-p}-\frac{p[1+1-2]}{2(1-p)} \Leftrightarrow h>\frac{1-p}{2} .
$$

Step 7. Consider the case in which $L>1-p$. We show that: $(1) \alpha_{H} \in(0,1),(2)$ $F_{L}(0) \in(0,1) ;(3) \alpha_{L} \in(0,1)$, and (4) the $L$-type places positive density at almost every $x \in(0, c]$.

Suppose $h \in(L / 2, L)$. First, we show that $\alpha_{H}<1$. Suppose $\alpha_{H}=1$. Then, $F_{L}(0)=1$. The $H$-type's expected payoff is $p$ if she bids arbitrarily close to zero, whereas her expected expected payoff is $(1-p) / 2+p-h<p$ if she bids $h$. Hence, she strictly prefers bidding $h$ to bidding any value below $h$, resulting in a contradiction.

Second, it is not possible that $\alpha_{L}>0=\alpha_{H}$, becasue the $L$-type knows that her opponent is the $H$-type and can profitably move weight down from $h$ to slightly above $c$.

Third, it is not possible that $\alpha_{L}=\alpha_{H}=0$. Suppose $\alpha_{L}=\alpha_{H}=0$. The $L$-type's expected payoff from bidding $h$ is $E U_{L}(h)=1-h / L>0$ if she bids $h$. So she has no incentive to bid 0 , and thus $F_{L}(0)=0$. On the other hand, we can show that $\underline{b}_{H}=0$. Suppose $\underline{b}_{H}>0$. The $L$-type will place zero density on almost every $x \in\left(0, \underline{b}_{H}\right]$, and thus $F_{L}\left(\underline{b}_{H}\right)=0$. Then, the $H$-type can profitably move density from $\left[\underline{b}_{H}, \underline{b}_{H}+\epsilon\right)$ down to slightly above 0 , resulting in a contradiction. Now, the $H$-type's expected payoff should be 0 because if the $H$-type bids arbitrarily close to zero, her expected payoff is 0 . However, if she bids $h$, her expeced utility is $E U_{H}(h)=1-h>0$, and thus she should 
have a mass at $h$, resulting in a contradiction. Hence, $\alpha_{H} \in(0,1)$.

Fourth, we show that $F_{L}(0)<1$. Because $\alpha_{H} \in(0,1)$, the $H$-type bids over $[0, c) \cup\{h\}$. If $F_{L}(0)=1$, following from equation 8 , we have for all $x \in[0, c]$,

$$
F_{H}(x)=\frac{x}{1-p}
$$

Then, if the $L$-type bidder bids $x \in[0, c]$, her the expected payoff is positive:

$$
E U_{L}(x)=F_{H}(x)-x / L=\frac{x}{1-p}-x / L>0 .
$$

Thus, the $L$-type can profitably move weight from 0 to above 0 , resulting in a contradiction. Hence, $\bar{b}_{L}>0$.

Fifth, it is not possible that $(1>) \alpha_{H}>0=\alpha_{L}$. In this case $h>c \geq \bar{b}_{L}>\underline{b}=0$. The $L$-type has the incentive to bid $h$ with a positive probability, because doing so leads to a higher expected payoff:

$$
\begin{aligned}
E U_{L}(h)-E U_{L}\left(\bar{b}_{L}\right) & =\left[F_{H}(c)+\frac{1-F_{H}(c)}{2}-\frac{h}{L}\right]-\left[F_{H}\left(\bar{b}_{L}\right)-\frac{\bar{b}_{L}}{L}\right] \\
& \geq\left[F_{H}\left(\bar{b}_{L}\right)+\frac{1-F_{H}\left(\bar{b}_{L}\right)}{2}-\frac{h}{L}\right]-\left[F_{H}\left(\bar{b}_{L}\right)-\frac{\bar{b}_{L}}{L}\right] \\
& =\left(h-\bar{b}_{L}\right)\left(\frac{1}{1-p}-\frac{1}{L}\right)-\frac{p\left[1+F_{L}\left(\bar{b}_{L}\right)-2 F_{L}(0)\right]}{2(1-p)}+\frac{p\left[F_{L}\left(\bar{b}_{L}\right)-F_{L}(0)\right]}{1-p} \\
& =\left(h-\bar{b}_{L}\right)\left(\frac{1}{1-p}-\frac{1}{L}\right)-\frac{p\left[1-F_{L}\left(\bar{b}_{L}\right)\right]}{2(1-p)} \\
& =\left(h-\bar{b}_{L}\right)\left(\frac{1}{1-p}-\frac{1}{L}\right) \\
& >0 .
\end{aligned}
$$

Hence, $\alpha_{L}$ should be positive, resulting in a contradiction.

Sixth, the $L$-type places positive density on almost every $x \in(0, c]$. Suppose not. Then, there exists $x, y$ such that $c \geq x>y \geq 0, F_{L}(y)>F_{L}(z)$ for all $z \in[0, y)$, and $F_{L}(x)=F_{L}(y)$,

$$
\begin{aligned}
E U_{L}(x)-E U_{L}(y) & =\left[F_{H}(x)-\frac{x}{L}\right]-\left[F_{H}(y)-\frac{y}{L}\right] \\
& =(x-y)\left(\frac{1}{1-p}-\frac{1}{L}\right)-\frac{p\left[F_{L}(x)-F_{L}(y)\right]}{1-p} \\
& =(x-y)\left(\frac{1}{1-p}-\frac{1}{L}\right) \\
& >0 .
\end{aligned}
$$

Thus, the $L$-type could profitably move density from at or below $y$ up to $x$. 
Proof of Proposition 3.2. In case 3, in which $h \leq L / 2$, following from Lemma 4.12 both bidders bid $h$ with a probability of one.

In cases 2.a and 3.a, in which $h \geq \max \{L, 1-p\}$, the cap is not restrictive and the equilibrium is the same as that of $\mathrm{Lu}$ et al. (2018).

The proof for cases 1.c in which $L<1-p$ and $h \in(L / 2,(1-p) / 2]$ is done in Lemma 4.13.i.b.

In the following, we consider the cases 1.b and 2.b, in which $h \in(\max \{L / 2,(1-$ $p) / 2\}, \max \{L, 1-p\})$.

For case 1.b, following from Lemma 4.13, we only need to solve a linear equation system and determine the values of $c$ and $F_{H}(c)$. The expected utility of the $H$-type is given below:

$$
p=E U_{H}(x)= \begin{cases}p+(1-p) F_{H}(x)-x & \text { for } x \in(0, c] \\ p+(1-p) F_{H}(c)+\frac{(1-p)\left[1-F_{H}(c)\right]}{2}-h & \text { for } x=h .\end{cases}
$$

We can then write out the following two equations with two unknowns:

$$
\begin{aligned}
(1-p) F_{H}(c)-c & =0 \\
(1-p) F_{H}(c)+\frac{(1-p)\left[1-F_{H}(c)\right]}{2}-h & =0 .
\end{aligned}
$$

Solving the above two equations with two unknowns yields

$$
\begin{aligned}
c & =2 h-(1-p) \\
F_{H}(c) & =\frac{2[h-(1-p) / 2]}{1-p} .
\end{aligned}
$$

In this case, the $H$-type bids according to a uniform distribution over $[0, c]$ with a probability of $F_{H}(c)$ and bids $h$ with a probability of $1-F_{H}(c)$, whereas the $L$-type bids 0 with a probability of 1 .

In case 2.b, following from Lemma 4.13, we only need to solve a linear equation system and determine the values of $c, F_{L}(0), F_{L}(c)$, and $F_{H}(c)$. The expected utilities of the $L$-type and the $H$-type are given, respectively, below:

$$
\begin{gathered}
0=E U_{L}(x)= \begin{cases}F_{H}(x)-\frac{x}{L} & \text { for } x \in(0, c] \\
F_{H}(c)+\frac{1-F_{H}(c)}{2}-\frac{h}{L} & \text { for } x=h ;\end{cases} \\
p F_{L}(0)=E U_{H}(x)= \begin{cases}p F_{L}(x)+(1-p) F_{H}(x)-x & \text { for } x \in(0, c] \\
p F_{L}(c)+(1-p) F_{H}(c)+\frac{p\left[1-F_{L}(c)\right]+(1-p)\left[1-F_{H}(c)\right]}{2}-h & \text { for } x=h .\end{cases}
\end{gathered}
$$


We can then write out the following four equations with four unknowns:

$$
\begin{gathered}
F_{H}(c)-\frac{c}{L}=0 ; \\
F_{H}(c)+\frac{1-F_{H}(c)}{2}-\frac{h}{L}=0 ; \\
p F_{L}(c)+(1-p) F_{H}(c)-c=p F_{L}(0) ; \\
p F_{L}(c)+(1-p) F_{H}(c)+\frac{p\left[1-F_{L}(c)\right]+(1-p)\left[1-F_{H}(c)\right]}{2}-h=p F_{L}(c) .
\end{gathered}
$$

Solving the above four equations with four unknowns yields

$$
\begin{aligned}
c & =2 h-L ; \\
F_{L}(0) & =\frac{1-L}{p} ; \\
F_{L}(c) & =\frac{2 h[L-(1-p)]}{p L}-1 ; \\
F_{H}(c) & =\frac{2(h-L / 2)}{L} .
\end{aligned}
$$

In this case, the $H$-type bids uniformly over $[0, c]$ with a probability of $F_{H}(c)$ and bids $h$ with a probability of $1-F_{H}(c)$; the $L$-type bids 0 with a probability of $F_{L}(0)$, bids uniformly over $[0, c]$ with a probability of $F_{L}(c)-F_{L}(0)$, and bids $h$ with a probability of $1-F_{L}(c)$.

\section{Appendix E: Revenue ranking}

Proof of Lemma 3.1. [i]. We first compare $E R^{C C D}$ and $E R^{C C C}$. Suppose $L>1-p$. Then, $E R^{C C D}<L<E R^{C C C}$ for all $h>L / 2$. Suppose $L \leq 1-p$. First, if $(1-p) / 2<$ $(1+p) L / 2$, then

$$
E R^{C C C}-E R^{C C D} \geq L-\left[p^{2} L+(1-p)^{2}\right]>L-\left[p^{2} L+(1-p)(1+p) L\right]=0
$$

Second, if $(1-p) / 2 \geq(1+p) L / 2$, then

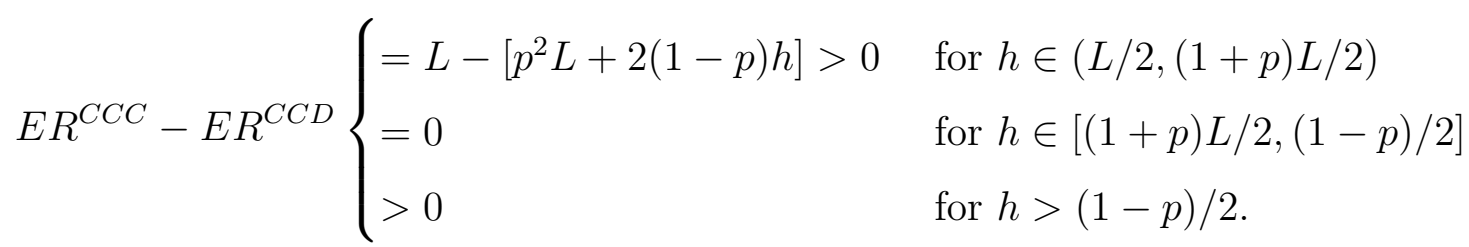

[ii]. We next compare $E R^{D C C}$ and $E R^{D D D}$. Suppose $2 p L \geq 1$. First, if $p>1 / 2$, then for $h \in(L / 2,1 / 2)$,

$$
E R^{D C C}-E R^{D D D} \geq L-\left[p^{2} L+(1-p)^{2} \cdot 2 h+2 p(1-p) \cdot \frac{L}{2}(1+L)\right]
$$




$$
\begin{aligned}
& \geq(1-p)\left[L-(1-p)-p L^{2}\right] \\
& \geq(1-p)\left[L-(1-p) \cdot 2 p L-p L^{2}\right] \\
& =(1-p)(1-L)[p(1+L)-1] \\
& =p+p L-1
\end{aligned}
$$

$>0$.

Second, if $2 p L \leq 1$, for $h \in(L / 2, \underline{x}]$,

$$
\begin{aligned}
E R^{D C C}-E R^{D D D} & =L-\left[p^{2} L+(1-p)^{2} \cdot 2 h+2 p(1-p) \cdot \frac{L}{2}(1+L)\right] \\
& \geq(1-p)\left[L-(1-p) L\left(\frac{p(1-L)}{1-p L}+1\right)-p L^{2}\right] \\
& \geq p^{2}(1-p) L(1-L)^{2} \cdot \frac{1}{1-p L} \\
& >0 .
\end{aligned}
$$

For $h>\underline{x}$,

$$
\begin{aligned}
E R^{D C C}-E R^{D D D} & =p(1-p) L-(1-p)^{2} L \cdot \frac{p(1-L)}{1-p L}-p(1-p) \cdot L^{2} \\
& =p^{2}(1-p) L(1-L)^{2} \cdot \frac{1}{1-p L} \\
& >0 .
\end{aligned}
$$

Let us now compare $E R^{D D D}$ and $E R^{C C D}$. First, suppose $L>1-p$.

$$
\begin{aligned}
E R^{D D D} & >\left[p^{2} L+(1-p)^{2} L+2 p(1-p) \cdot \frac{L}{2}(1+L)\right] \\
& =p L+(1-p)^{2} L+p(1-p) L \cdot L \\
& >p L+(1-p)^{2} L^{2}+p(1-p) L^{2} \\
& =[p+(1-p) \cdot L] L \\
& =E R^{C C D}
\end{aligned}
$$

Second, suppose $L \leq 1-p$. For $h \in(L / 2,(1-p) / 2)$,

$$
\begin{aligned}
E R^{D C C}(h)-E R^{D D D}(h) & =p(1-p)[-2 h+L(1+L)] \\
& \geq p(1-p)\left[L-(1-p)+L^{2}\right] \\
& \geq p(1-p) \cdot L^{2} \\
& >0 .
\end{aligned}
$$


For $h \geq(1-p) / 2, E R^{D C C}(h)-E R^{D D D}(h) \geq E R^{D C C}\left(\frac{1-p}{2}\right)-E R^{D D D}\left(\frac{1-p}{2}\right)>0$. 